\title{
Coloração de Grafos e o Método Probabilístico
}

\author{
DANiEL Morgato Martin
}

DISSERTAÇÃO APRESENTADA

$\mathrm{AO}$

INSTITUTO DE MATEMÁTICA E ESTATÍSTICA

DA

UNIVERSIDADE DE SÃO PAULO

PARA

A OBTENÇÃO DO GRAU DE MESTRE

$\mathrm{EM}$

CIÊNCIA DA COMPUTAÇÃO

Orientador: Prof. Dr. Yoshiharu Kohayakawa

Durante o mestrado o autor teve bolsa da FAPESP, proc. \#03/12046-3 


\section{Coloração de Grafos e o Método Probabilístico}

Este exemplar corresponde à redação final da tese devidamente corrigida e defendida por Daniel Morgato Martin e aprovada pela comissão julgadora.

São Paulo, 15 de agosto de 2005

Banca examinadora:

Prof. Dr. Yoshiharu Kohayakawa (orientador) Prof. Dr. Paulo Feofiloff

Prof. Dr. Carlos Gustavo Tamm de A. Moreira Profa. Dra. Yoshiko Wakabayashi (suplente) Prof. Dr. Jozef Skokan (suplente)
IME-USP

IME-USP

IMPA

IME-USP

U. of Illinois 
À minha família

ii 


\section{Agradecimentos}

Agradeço a Deus por todo o bem que me concedeu.

Agradeço a toda a minha família; especialmente aos meus pais pela educação que recebi e aos meus irmãos por todos os incentivos.

Agradeço ao Yoshi, meu orientador, pela grande ajuda e atenção que me dispensou, e por todas as oportunidades que me deu. Não poderia esquecer das professoras Yoshiko e Cris, que também me apoiaram muito.

Muito obrigado ao professor Paulo F. que leu atentamente a minha dissertação, apontou suas falhas e deu boas sugestões de mudança. Também agradeço ao Marcel e ao Cardonha pelas correções que fizeram.

Agradeço pela companhia dos meus colegas de turma e amigos: Fernando Mario, Daniel Cordeiro, Daniel Vaqueiro, Marcos Broinizi, João Soares, ao Stefan e à Flávia.

Aos amigos: Gordana, Marcel, Cristiane, Jozef, Cardonha, Domingos, Juliana, Marcelo, Elen, Said e Felipe.

Àqueles de que me esqueci no momento, peço desculpas.

Muito obrigado a todos! 


\section{Resumo}

Nesta dissertação estudamos alguns problemas envolvendo coloração de grafos, e focamos em alguns resultados a respeito desse assunto que usam o método probabilístico.

Vamos, primeiramente, demonstrar o Teorema de Brooks e o Teorema de Vizing, que são os dois primeiros resultados que qualquer estudante da área vê a respeito de coloração de vértices e arestas respectivamente. Em seguida, introduzimos o conceito de lista-coloração e mostramos uma prova do Teorema de Galvin, que até recentemente era um problema em aberto. O Teorema de Galvin afirma que para qualquer grafo bipartido $G$, o número cromático e o número lista-cromático são iguais. Ainda na primeira parte do texto, explicamos o que é coloração total e enunciamos a principal conjectura que existe a respeito desse assunto.

Depois disso, numa segunda parte do texto, fazemos um resumo de conceitos probabilísticos e de algumas ferramentas como o Lema Local e algumas desigualdades importantes. Esses conceitos são usados no restante do texto.

Em seguida, mostramos algumas aplicações do método probabiístico para resolver problemas de lista-coloração e problemas de coloração total.

Por último, mostramos um teorema de Kim que usa o método semialeatório de Rödl para provar o seguinte: para todo $\epsilon>0$ existe $\Delta_{0}$ tal que para todo grafo $G$ com cintura pelo menos 5 e grau máximo $\Delta \geq \Delta_{0}$, tem-se $\chi(G) \leq(1+\epsilon) \Delta / \ln \Delta$. 


\section{Abstract}

In this thesis we study some problems on graph colorings, focussing on some applications of the probabilistic method to tackle them.

We start by showing Brooks's theorem and Vizing's theorem, which are the first results almost every student of the area starts with. Next, we introduce the concept of list-coloring and we give a proof of Galvin's theorem, which is a relatively recent result. Galvin's result states that for every bipartite graph $G$, the chromatic number and the choosability number are the same. Still in the first part of this text, we explain what total colorings are and we state the main conjecture involving such colorings.

In the second part of the text, we discuss some probabilistic concepts and tools, such as the Local Lema and some important inequalities. These concepts are used in the remainder of the thesis.

In what follows, we show some applications of the probabilistic method to solve list-coloring and total coloring problems.

Finally, we go into the details of a theorem of Kim about graphs with girth at least 5 . The proof of this theorem makes use of the semi-random method, due to V. Rödl. Kim's theorem asserts that for every $\epsilon>0$ there is $\Delta_{0}$ such that, for every graph with girth at least 5 and maximum degree $\Delta \geq \Delta_{0}$, we have $\chi(G) \leq(1+\epsilon) \Delta / \ln \Delta$. 


\section{Sumário}

1 Introdução 1

2 Definições básicas e notação 2

3 Resultados básicos em coloração 5

3.1 Coloração de vértices . . . . . . . . . . . . . . . 5

3.2 Coloração de arestas . . . . . . . . . . . . . . . . . . . 8

3.3 Lista-coloração . . . . . . . . . . . . . . . . . . . . . . . . . . . 10

3.4 Coloração total . . . . . . . . . . . . . . . . . . . . . . . . 14

4 Ferramentas probabilísticas $\quad 15$

4.1 Conceitos básicos . . . . . . . . . . . . . . . . . . . 15

4.2 Método do Primeiro Momento . . . . . . . . . . . . . . . . . . 17

4.3 O Lema Local . . . . . . . . . . . . . . . . . . . . . . . . . . . 18

4.4 Ferramentas de concentração . . . . . . . . . . . . . . . 21

5 Resultados probabilísticos em coloração $\quad 25$

5.1 Número lista cromático . . . . . . . . . . . . . . . . . . . . 25

5.1.1 Uma conjectura de K. Ohba . . . . . . . . . . . . 30

5.2 Coloração total de grafos . . . . . . . . . . . . . . . . . 34

6 Grafos Livres de Triângulos $\quad 38$

6.1 Introdução . . . . . . . . . . . . . . . . . . . . 38

6.2 Uma intuição . . . . . . . . . . . . . . . . . . . . . . . 39

6.3 Uma cota fraca . . . . . . . . . . . . . . . . . . . . . . 42

6.4 O Teorema de J. H. Kim . . . . . . . . . . . . . . . 47

6.4.1 Construindo o supergrafo regular . . . . . . . . . . . . . . . 47

6.4.2 O procedimento . . . . . . . . . . . . . 48 
6.4 .3 Objetivos . . . . . . . . . . . . . . . . . . . 50

6.4 .4 Parâmetros de interesse . . . . . . . . . . . . . . 50

6.4.5 Calculando algumas probabilidades . . . . . . . . . . 51

6.4.6 Visão geral do restante da prova . . . . . . . . . . . . 52 


\section{Capítulo 1}

\section{Introdução}

Coloração de grafos foi um assunto muito estudado no século passado devido à vontade existente, na época, de se encontrar uma solução para a conjectura das 4 cores. Graças a isso, a área de coloração de grafos é muito vasta atualmente. Existem diversas questões de interesse muito estudadas que, na verdade, são variantes do problema clássico de colorir propriamente os vértices de um grafo. Uma idéia bem natural é colorir as arestas de um grafo sem que duas adjacentes tenham a mesma cor. Podemos também pensar em colorir vértices (ou arestas) restringindo o conjunto de cores permitidas em cada vértice (aresta). Essa variante encaixa-se no que chamamos de coloração restrita. Outra variante é colorir vértices e arestas sem que elementos adjacentes ou incidentes tenham a mesma cor. Chamamos essa variante de problema da coloração total.

Atualmente, existem várias questões importantes que ainda não foram resolvidas. Nesta monografia, pretendemos apresentar os resultados clássicos da área, com enfoque no problema da lista-coloração e da coloração total. Muitos desses resultados apontam na direção de que as conjecturas são verdadeiras. Nas demonstrações de alguns teoremas, faremos uso do método probabilístico criado por P. Erdős (ver [2]). Um apanhado das técnicas probabilísticas utilizadas é feito no capítulo Ferramentas probabilísticas.

O texto está dividido em três partes principais, sendo elas: resultados básicos em coloração, resultados probabilísticos em coloração e grafos livres de triângulos. A primeira parte do texto apresenta, muito sucintamente, alguns resultados clássicos da área de coloração de grafos. As outras duas apresentam diversos resultados relevantes que foram construídos com o uso do método probabilístico. 


\section{Capítulo 2}

\section{Definições básicas e notação}

Muitos termos e símbolos que vamos descrever aqui são bem conhecidos do público que provavelmente tem interesse em ler este texto. No entanto, para ter certeza de que usamos a mesma linguagem, vamos enumerar a nomenclatura necessária para a leitura do texto.

Todos os conjuntos que aparecem neste texto são finitos. Se $X$ é um conjunto, denotamos o número de elementos de $X$ por $|X|$. Se $X$ é um conjunto com $k$ elementos, dizemos que $X$ é um $k$-conjunto. O conjunto de todos os $k$-subconjuntos de um conjunto $X$ é denotado por $\left(\begin{array}{l}X \\ k\end{array}\right)$. O conjunto dos $k$ primeiros inteiros positivos é denotado por $[k]$.

Um grafo é um par ordenado $G=(V, E)$, onde $V$ é o conjunto dos vértices e $E \subseteq\left(\begin{array}{c}V \\ 2\end{array}\right)$ é o conjunto das arestas. Novamente, todos os grafos considerados aqui são finitos, isto é, possuem um conjunto finito de vértices. Se e é uma aresta e $u \in e$, dizemos que $u$ incide em $e$ ou, equivalentemente, $e$ incide em $u$. Também dizemos que $u$ é uma ponta de $e$. Se $u, v \in e$ para algum $e \in E$, dizemos que $u$ e $v$ são vértices adjacentes, ou vizinhos, ou ainda, que $u$ e $v$ estão ligados pela aresta $e$. Denotamos uma aresta $e=\{u, v\}$ simplesmente escrevendo uv ou vu. Duas arestas e e $f$ são adjacentes se possuem alguma ponta em comum.

Se $X \subseteq V$ é um subconjunto de vértices do grafo, denotamos por $\nabla(X)$ o conjunto de arestas que têm uma ponta em $X$ e outra em $V \backslash X$. Vamos denotar a cardinalidade de $\nabla(X)$ por $d(X)$. Às vezes, abusamos da notação e escrevemos $\nabla(v)$ para denotar o conjunto de arestas incidentes em $v$. Nesse caso, $d(v)$ é chamado grau de $v$. Escrevemos $\Delta$ para denotar o grau máximo do grafo $\left(\Delta=\max _{v \in V} d(v)\right)$, e $\delta$ para denotar o grau mínimo. Se $X \subseteq V$ é um subconjunto de vértices do grafo, denotamos por $\Gamma(X)$ o conjunto dos 
vértices que são adjacentes a algum vértice de $X$. Note que $\Gamma(X)$ não é necessariamente disjunto de $X$. Abusando da notação, escrevemos $\Gamma(v)$ para denotar o conjunto de vizinhos de $v$.

Um grafo $G^{\prime}=\left(V^{\prime}, E^{\prime}\right)$ é subgrafo de um grafo $G=(V, E)$ se $V^{\prime} \subseteq V$ e $E^{\prime} \subseteq E$. Quando isso ocorre, podemos escrever $G^{\prime} \subseteq G$. Um subgrafo de $G$ é próprio se alguma das continências acima for própria. Quando houver ambigüidade, vamos escrever os conjuntos/invariantes definidos acima mencionando a que grafo se referem. Por exemplo, diremos $V(G)$ em vez de $V$, $E(G)$ em vez de $E$, e assim por diante: $\Delta(G), \delta(G), \nabla_{G}(v), d_{G}(v), \Gamma_{G}(v)$, etc.

Um subgrafo $H$ de $G$ é induzido por um conjunto de vértices $X$, se $V(H)=X$ e $E(H)=E(G) \cap\left(\begin{array}{c}X \\ 2\end{array}\right)$. Para nós, um subgrafo $H$ de $G$ é gerado por um conjunto de arestas $M$, se

$$
V(H)=\bigcup_{f \in M} f \quad \text { e } E(H)=M .
$$

Para denotar o subgrafo induzido (gerado) por um conjunto $X$ de vértices (arestas), escrevemos $G[X]$. Se $X$ é um subconjunto de vértices de um grafo $G$, então $G-X$ denota o subgrafo obtido removendo-se os vértices de $X$ e as arestas incidentes a eles, isto é, o subgrafo de $G$ induzido por $V(G) \backslash X$. Se $X=\left\{v_{1}, \ldots, v_{k}\right\}$, às vezes escrevemos $G-v_{1}-\cdots-v_{k}$ em vez de $G-X$.

O grafo linha de um grafo $G$, denotado por $L(G)$, é um grafo onde $V(L(G))=E(G)$, e $\{e, f\}$ é uma aresta de $L(G)$ se e só se $e$ e $f$ forem arestas adjacentes em $G$. Grafos linha surgirão naturalmente quando falarmos de coloração de arestas.

Dizemos que dois grafos $G$ e $H$ são isomorfos se existe uma bijeção $f: V(G) \longrightarrow V(H)$ tal que $f(x) f(y) \in E(H)$ se e só se $x y \in E(G)$. Para denotar que $G$ e $H$ são isomorfos escrevemos $G \cong H$. Dizemos que um subgrafo $G^{\prime}$ de um grafo $G$ é um " $H$ em $G$ " se $G^{\prime} \cong H$.

Um caminho é um grafo $P$ com conjunto de vértices $V(P)=\left\{v_{1}, \ldots, v_{k}\right\}$ e com conjunto de arestas $E(P)=\left\{v_{i} v_{i+1}: i \in[k-1]\right\}$. Dizemos que $v_{1}$ e $v_{k}$ são pontas de $P$.

$\operatorname{Um}(u, v)$-caminho em $G$ é um caminho cujas pontas são $u$ e $v$. Um grafo $G$ é conexo se, para qualquer par de vértices distintos $u, v \in V(G)$, existe um $(u, v)$-caminho em $G$. Um grafo é $k$-conexo se a remoção de menos que $k$ vértices não desconecta o grafo. Um grafo é $k$-aresta-conexo se a remoção de menos que $k$ arestas não desconecta o grafo. Um conjunto de vértices 
$X \subseteq V(G)$ é um conjunto de corte, se a remoção de $X$ desconecta $G$. Se $G$ é desconexo, então $\emptyset$ é um conjunto de corte. Um bloco de um grafo $G$ é um subgrafo 2-conexo maximal de $G$, isto é, não é subgrafo de nenhum outro subgrafo 2-conexo de $G$.

Um circuito é um grafo conexo que possui todos os vértices com grau exatamente 2. Um grafo $G$ é completo se $E(G)=\left(\begin{array}{c}V(G) \\ 2\end{array}\right)$. Um subgrafo completo de um grafo $G$ é chamado clique. O tamanho do maior clique de um grafo $G$ é denotado por $\omega(G)$. Um conjunto $X$ de vértices de um grafo $G$ é estável, ou independente, se $E(G[X])=\emptyset$. O tamanho do maior conjunto independente de um grafo $G$ é denotado por $\alpha(G)$. Um emparelhamento em um grafo $G$ é um conjunto de arestas duas a duas não adjacentes.

Sejam $f$ e $g$ duas funções. Escrevemos $f=O(g)$ se $f \leq c g$, para alguma constante absoluta $c$. Escrevemos $f=\Omega(g)$ se $g=O(f)$, e $f=\Theta(g)$ se $f=O(g)$ e $f=\Omega(g)$. Quando o limite de $f / g$ tende a zero, escrevemos $f=$ $o(g)$. Quando escrevemos "log", está subentendido que a base do logaritmo é 2. Para denotar que a base do logaritmo é a base natural, escrevemos "In".

Se alguma notação utilizada não se encontra aqui, ela é conforme a notação de [10]. 


\section{Capítulo 3}

\section{Resultados básicos em coloração}

\subsection{Coloração de vértices}

Uma coloração dos vértices (ou, simplesmente, coloração) de um grafo $G$ é uma atribuição de cores aos seus vértices (formalmente é uma função $\varphi: V(G) \longrightarrow C$, onde $C$ é um conjunto de cores). Dizemos que uma coloração é própria se vértices adjacentes recebem cores diferentes. Uma $k$-coloração é uma coloração que usa até $k$ cores (geralmente os inteiros de 1 até $k$ ). Um grafo $G$ é $k$-colorível se admite uma $k$-coloração própria.

Para cada cor $c, \varphi^{-1}(c)$ é dita ser uma classe de coloração. Suponha que o conjunto de cores usadas é $C=[k]$. Toda $k$-coloração própria $\varphi$ induz naturalmente uma partição $\left\{\varphi^{-1}(1), \ldots, \varphi^{-1}(k)\right\}$ do conjunto de vértices do grafo em conjuntos estáveis. Por essa razão, um grafo $k$-colorível também é chamado de $k$-partido. Quando $k=2$, dizemos bipartido em vez de 2 partido. Para explicitar as classes $A$ e $B$ de uma coloração própria de um grafo bipartido, dizemos que o grafo é $(A, B)$-bipartido.

O número cromático de um grafo $G$, denotado por $\chi(G)$, é o menor inteiro $k$ tal que $G$ é $k$-colorível. Dizemos que um grafo $G$ é $k$-cromático se $\chi(G)=k$.

Um limitante superior fácil para o número cromático de um grafo é $\chi(G) \leq \Delta(G)+1$. Para vermos isso, suponha que já colorimos vários vértices do grafo, e suponha que estamos tentando colorir um certo vértice $v$. Precisamos colocar uma cor em $v$ que seja diferente das cores dadas aos vizinhos de $v$ (ignore os vizinhos que ainda não foram coloridos). Temos $\Delta(G)+1$ cores possíveis para dar a $v$ e no máximo $\Delta(G)$ cores a evitar. Assim, é possível escolher para $v$ uma cor que seja diferente das cores dadas aos seus 
vizinhos. Este procedimento pode ser repetido até que todos os vértices do grafo estejam coloridos. Portanto temos que $\chi(G) \leq \Delta(G)+1$. O método para colorir grafos que acabamos de descrever chama-se método da coloração gulosa.

O número de coloração de $G$ é definido como sendo $\hat{\delta}(G)+1$, onde $\hat{\delta}(G)$ é o máximo dentre os graus mínimos de todos os subgrafos induzidos de $G$. O resultado do parágrafo anterior pode ser levemente melhorado pondo-se $\hat{\delta}(G)+1$ em vez de $\Delta(G)+1$. Considere uma ordenação $v_{1}, \ldots, v_{n}$ dos vértices de $G$ de forma que $v_{i}$ é um vértice de grau mínimo em $G\left[\left\{v_{1}, \ldots, v_{i}\right\}\right]$. Executando o método da coloração gulosa, seguindo esta dada ordem dos vértices, temos que apenas $\hat{\delta}(G)+1$ cores são necessárias. Isso pode não fazer diferença em alguns casos, por exemplo, quando $G$ é um grafo regular. Nesse caso $\hat{\delta}(G)=\Delta(G)$.

A seguir, veremos um teorema que diminui de 1 a cota do número cromático quando o grafo satisfaz algumas modestas condições.

Lema 1: Todo grafo 2-conexo $G$, com grau máximo pelo menos 3, que não é completo, contém três vértices $x, y, z$ com $x y, x z \in E(G), y z \notin E(G)$, e $G-y-z$ é conexo.

Prova. Seja $x$ um vértice de grau máximo em $G$. Dois de seus vizinhos não estão ligados por uma aresta. Caso contrário, se todos os vizinhos de $x$ estivessem ligados entre si, como $G$ não é completo, algum vizinho de $x$ deveria ter grau maior que $d(x)$ : isso é absurdo, pois $d(x)=\Delta(G)$. Se $x$ é adjacente a todos os demais vértices do grafo $G$, tome $y$ e $z$ como sendo vizinhos de $x$ que não estão ligados entre si, e acabou. Suponha então que $x$ não é adjacente a todos os demais vértices de $G$. Então existem $v$ e $w$ tais que $v$ é vizinho de $x$, e $w$ é vizinho de $v$ mas não de $x$. Se $G-x-w$ é conexo, então estamos feitos. Suponha então que $\{x, w\}$ seja um conjunto de corte.

Sejam $C_{1}, \ldots, C_{s}$ as componentes de $G-x-w$. Note que $s \geq 2$. Claramente $G-w$ é conexo, pois $G$ é 2-conexo. Isso implica que $x$ tem um vizinho em cada $C_{i}$. Sejam $y$ e $z$ dois vizinhos de $x$ que estão em $C_{1}$ e $C_{2}$ respectivamente. Tome $G^{\prime}=G-x$. Sejam $B_{y}$ e $B_{z}$ os blocos 2-conexos de $G^{\prime}$ que contém $y$ e $z$ respectivamente. Claramente $B_{y} \neq B_{z}$.

Os vértices $y$ e $z$ não são adjacentes pois estão em componentes diferentes de $G-x-w$. A remoção de $y$ não desconecta $B_{1}$. A remoção de $z$ não desconecta $B_{2}$. Como $y$ e $z$ são diferentes de $w, G^{\prime}-y-z$ é conexo. (É preciso refletir um pouco para ver que essa afirmação é verdadeira.) Gostaríamos de 
afirmar que a remoção de ambos também não desconecta $G$, porém devemos ser cautelosos. O vértice $x$ poderia ser um vértice isolado em $G-y-z$, acontece que $x$ tem grau pelo menos 3 e, portanto, $G-y-z$ é conexo como gostaríamos.

Lema 2: Seja $G$ um grafo 2-conexo que tem grau máximo pelo menos 3 e que não é completo. Podemos colocar os vértices de $G$ em uma ordem $v_{1}, \ldots, v_{n}$ de tal forma que $v_{1} v_{2} \notin E(G), v_{1} v_{n}, v_{2} v_{n} \in E(G)$, e para todo $j$, $3 \leq j \leq n-1$, $v_{j}$ tem no máximo $\Delta(G)-1$ vizinhos em $\left\{v_{1}, \ldots, v_{j-1}\right\}$.

Prova. Tome $x, y$ e $z$ como no lema. Ponha $v_{1}=y, v_{2}=z$ e $v_{n}=x$. Pelo Lema 1 , sabemos que $G-v_{1}-v_{2}$ é conexo. Ordene os vértices de $G-v_{1}-v_{2}$ da seguinte forma: no primeiro passo escolha um vértice $u$ que seja adjacente a $v_{n}$ e ponha $v_{n-1}=u$. No passo $i, 1<i<n-2$, escolha $u$ que seja adjacente a alguém de $v_{n}, v_{n-1}, \ldots, v_{n-i+1}$ e ponha $v_{n-i}=u$. É fácil ver que a ordem descrita acima possui as propriedades desejadas.

Teorema 3 (Brooks): Para todo grafo $G$ com grau máximo $\Delta, \chi(G) \leq \Delta$ a menos que alguma componente de $G$ seja um clique com $\Delta+1$ vértices ou $\Delta=2$ e alguma componente de $G$ seja um circuito ímpar.

Prova. É fácil verificar que o teorema vale se $\Delta=2$. Suponha $\Delta \geq 3$. Seja $G$ um contra-exemplo com o menor numero possível de vértices. Afirmamos que $G$ deve ser 2-conexo. É fácil ver que $G$ deve ser conexo. Para provar que $G$ é 2-conexo, suponha, por contradição, que exista um vértice de corte $v$ em $G$, e seja $C$ uma componente de $G-v$. Ponha $G_{1}:=G[V(C) \cup\{v\}]$ e $G_{2}:=G-C$. Observe que tanto $G_{1}$ como $G_{2}$ não são cliques com $\Delta+1$ vértices. Se $G_{1}$ (ou $G_{2}$ ) for um circuito ímpar, podemos usar $3 \leq \Delta$ cores para colori-lo. Agora, colorimos $G_{1}$ e $G_{2}$ separadamente, usando no máximo $\Delta$ cores em cada um deles, e ajustamos a cor de $v$ para que seja a mesma nas duas partes.

Basta provar o teorema para grafos 2-conexos com $\Delta \geq 3$. Suponha, então, que $G$ seja um grafo 2-conexo com $\Delta \geq 3$ e suponha que $G$ não seja um clique com $\Delta+1$ vértices. Considere uma ordenação dos vértices de $G$ como no Lema 2. Se aplicarmos o método da coloração gulosa seguindo esta ordem dos vértices, então podemos colorir $v_{1}$ e $v_{2}$ com a mesma cor. Para cada vértice $v_{i}, 2<i<n$, podemos colori-lo usando apenas cores de 1 a $\Delta$, pois temos no máximo $\Delta-1$ cores a evitar. O vértice $v_{n}$ tem $\Delta$ vizinhos 
já coloridos, mas $v_{1}$ e $v_{2}$ têm a mesma cor. Então podemos usar, também, somente cores de 1 a $\Delta$ para colori-lo.

\subsection{Coloração de arestas}

Uma coloração das arestas de um grafo $G$ é uma atribuição de cores às suas arestas (formalmente é uma função $\varphi: E(G) \longrightarrow C$, onde $C$ é um conjunto de cores). Dizemos que uma colorão de arestas é própria se arestas adjacentes recebem cores diferentes. O indice cromático de um grafo $G$, denotado por $\chi^{\prime}(G)$, é o menor inteiro $k$ tal que existe uma coloração própria das arestas de $G$ usando $k$ cores. Note que colorir as arestas de um grafo $G$ é o mesmo que colorir os vértices do grafo linha $L(G)$.

Gostaríamos de mencionar que os resultados a seguir que envolvem coloração de arestas são, na verdade, um pouco mais gerais. Fazendo ligeiras modificações em seus enunciados e demonstrações, eles seriam verdadeiros também para multigrafos. Num multigrafo, o conjunto de arestas não é propriamente um conjunto, mas sim um multiconjunto, no qual a multiplicidade dos seus elementos é levada em conta.

Se tentássemos colorir propriamente as arestas de um grafo $G$, perceberíamos que são necessárias pelo menos $\Delta(G)$ cores. Um limitante superior natural que poderíamos tomar para $\chi^{\prime}(G)$ é $\Delta(L(G))+1 \leq 2 \Delta(G)-1$. O teorema abaixo, no entanto, reduz bem o limitante, e mostra que $\chi^{\prime}(G)$ é bem próximo de $\Delta(G)$. Na verdade, o índice cromático é $\Delta(G)$ ou $\Delta(G)+1$.

Teorema 4 (Vizing): Para todo grafo $G$, temos $\chi^{\prime}(G) \leq \Delta(G)+1$.

Prova. Vamos provar o teorema por indução no número de arestas. Se $G$ não tem arestas ou tem exatamente uma aresta, a afirmação é verdadeira. Suponha, então, que $G$ tem pelo menos duas arestas e suponha que o resultado seja válido para grafos com menos arestas do que $G$. Por hipótese de indução, podemos supor que todas as arestas de $G$ estão coloridas com $\Delta(G)+1$ cores, exceto uma aresta $e=x y_{1}$ que vamos tentar colorir.

Dizemos que uma cor $c$ está livre em um vértice $v$, se nenhuma aresta que incide em $v$ foi colorida com a cor $c$. Observe que em cada vértice, pelo menos uma cor está livre. O que vamos fazer é construir uma seqüência de vértices $y_{1}, y_{2}, \ldots, y_{k}$ adjacentes a $x$, e uma seqüência de cores $t_{1}, t_{2}, \ldots, t_{k}$ tais que a cor $t_{i}$ está livre no vértice $y_{i}$, mas a aresta $x y_{i+1}$ tem cor $t_{i}$. Suponha que já tenhamos os vértices $y_{1}, y_{2}, \ldots, y_{j}$ e as cores $t_{1}, t_{2}, \ldots, t_{j}$. No máximo 
uma aresta $x y$ possui cor $t_{j}$. Se existe tal aresta, então tomamos $y_{j+1}=y \mathrm{e}$ tomamos uma cor $t_{j+1}$ que está livre em $y_{i+1}$.

Há dois motivos pelos quais somos obrigados a parar de construir essas seqüências:

(i) Não existe aresta incidente a $x$ com cor $t_{k}$. Nesse caso, colorimos cada aresta $x y_{i}$ com cor $t_{i}$, para $i<k$. E agora, como $t_{k}$ está livre tanto em $x$ como em $y_{k}$, podemos colorir a aresta $x y_{k}$ com cor $t_{k}$. Neste caso, podemos parar, pois já atingimos nosso objetivo.

(ii) Existe algum índice $j<k$ tal que $x y_{j}$ possui cor $t_{k}$. Caso isso aconteça, pinte cada aresta $x y_{i}$ com cor $t_{i}$, para $i<j$. Nesse ponto, a aresta sem cor é a aresta $x y_{j}$. Discutiremos este caso no próximo parágrafo.

Temos então uma seqüência $y_{j}, \ldots, y_{k}$ e cores $t_{j}, \ldots, t_{k}$, onde a cor $t_{i}$ está livre em $y_{i}$ e está sendo usada em $y_{i+1}$. Ademais, $x y_{j}$ não está colorido com nenhuma cor e $t_{k}$ está livre em $y_{j}$ e em $y_{k}$. Seja $s$ uma cor livre em $x$. Se $s$ está livre em $y_{t}$, para algum $j \leq t \leq k$, então colorimos a aresta $x y_{i}$ com cor $t_{i}$, para $j \leq i<t$, e colorimos $x y_{t}$ com a cor $s$. E, portanto, teríamos colorido o grafo todo. Caso $s$ não esteja livre em $y_{t}$, para $j \leq t \leq k$, então considere o subgrafo $H \subseteq G$ gerado pelas arestas de cor $s$ e de cor $t_{k}$. Todos os vértices de $H$ têm grau no máximo dois (pois cada um incide, no máximo, numa aresta de cor $s$ e numa de cor $t_{k}$ ). Então as componentes de $H$ só podem ser circuitos ou caminhos. Mas $x, y_{j}$ e $y_{k}$ têm grau no máximo $1 \mathrm{em}$ $H$, e portanto uma das duas alternativas abaixo ocorre:

(i) $x$ e $y_{j}$ estão em componentes diferentes de $H$. Nesse caso, troque a cor $s$ pela cor $t_{k}$, nas arestas da componente de $H$ que contém $y_{j}$. Assim $s$ será uma cor livre em $x$ e $y_{j}$, e portanto podemos atribuir cor $s$ à aresta $x y_{j}$ que faltava ser colorida.

(ii) $x$ e $y_{k}$ estão em componentes diferentes de $H$. Nesse caso, continue a colorir as arestas $x y_{i}$ com a cor $t_{i}, j \leq i<k$. Agora a aresta não colorida é a aresta $x y_{k}$. Troque a cor $s$ pela cor $t_{k}$ nas arestas da componente de $H$ que contém $y_{k}$. Assim $s$ será uma cor livre em $x$ e $y_{k}$, e portanto podemos atribuir cor $s$ à aresta $x y_{k}$ que faltava ser colorida.

Com isso, a prova do teorema está completa. 
Fig. 1: Ambos os grafos possuem grau máximo 3, porém uma coloração das arestas do grafo da esquerda necessita apenas de 3 cores, enquanto que, para colorir as arestas do grafo da direita, precisamos de 4 cores.

\subsection{Lista-coloração}

Seja $G$ um grafo. Agora, vamos dar a cada vértice $v \in V(G)$ um conjunto $\mathcal{L}(v)$ de cores permitidas. Na literatura, esses conjuntos são chamados de listas, mas a ordem das cores nessas "listas" não importa. Dizemos que $G$ é $\mathcal{L}$-colorível se existe uma coloração própria $\varphi$ tal que $\varphi(v) \in \mathcal{L}(v)$, para todo vértice $v$. O número lista-cromático, denotado por $c h(G)$, é o menor inteiro $k$ tal que, quaisquer que sejam as listas atribuidas aos vértices, se todas elas têm tamanho pelo menos $k$, então $G$ é $\mathcal{L}$-colorível. É óbvio que $\operatorname{ch}(G)$ é pelo menos tão grande quanto o número cromático:

$$
\operatorname{ch}(G) \geq \chi(G)
$$

Mas não é óbvio que ele pode ser, como de fato é em muitos casos, muito maior que o número cromático. Na verdade, isso é totalmente contra a nossa intuição. Se pensarmos que $\mathcal{L}=\{\mathcal{L}(v)=\{1, \ldots, \chi(G)\}: v \in V(G)\}$ é um conjunto de listas "viável", então por que um outro conjunto de listas, todas elas com $\chi(G)$ cores, permitindo até mais cores do que simplesmente $\{1, \ldots, \chi(G)\}$, não seria "viável"? A construção de um contra-exemplo é razoavelmente simples, como podemos observar na figura abaixo. 
O grafo desenhado não possui uma coloração própria que respeita as listas indicadas.

Mais genericamente, podemos construir grafos bipartidos com número lista-cromático arbitrariamente alto. Seja $G$ um grafo $(A, B)$-bipartido completo com $|A|=|B|=\left(\begin{array}{c}2 k-1 \\ k\end{array}\right)$. Ponha cada $k$-subconjunto de $\{1,2, \ldots, 2 k-1\}$ como sendo a lista de algum $a \in A$ e de algum $b \in B$. Dessa forma, pelo menos $k$ cores são usadas em qualquer coloração dos vértices de $A$ e pelo menos $k$ cores são usadas em qualquer coloração dos vértices de $B$. Uma cor aparece, então, em ambas as partes e portanto $G$ não é $\mathcal{L}$-colorível.

Embora o número lista-cromático não esteja relacionado com o número cromático, o índice lista-cromático (definido analogamente) parece estar intimamente ligado com o índice cromático.

Conjectura 1 (Conjectura da Lista-Coloração): Para todo grafo G, é verdade que $\operatorname{ch}^{\prime}(G)=\chi^{\prime}(G)$.

A conjectura é verdadeira para grafos bipartidos como mostra o Teorema de Galvin abaixo. Para apresentá-lo aqui, precisaremos apresentar o conceito de emparelhamentos estáveis em grafos bipartidos. A prova do teorema de Galvin aqui apresentada foi encontrada em [4].

Seja $G$ um grafo $(A, B)$-bipartido. Considere uma função preferência $\mu_{v}: \Gamma(v) \longrightarrow \mathbb{N}$ para cada vértice $v$ do grafo. Suponha que $\mu_{v}$ é injetora para todo $v$. Se $x, y$ são vizinhos de $v$ e $\mu_{v}(x)>\mu_{v}(y)$, dizemos que $v$ prefere $x$ a $y$. Se $u$ é um vértice e $M$ é um emparelhamento que contém uma aresta $e$ incidente em $u$, vamos escrever $M(u)$ para denotar a outra ponta de $e$. Um emparelhamento $M$ de $G$ é estável se, para cada aresta $u v \in E(G)-M$, temos que alguma aresta de $M$ incide em $u$ e $\mu_{u}(M(u))>\mu_{u}(v)$, ou que alguma aresta de $M$ incide em $v$ e $\mu_{v}(M(v))>\mu_{v}(u)$. Não é claro que para qualquer grafo bipartido existe um emparelhamento estável. O Teorema de Gale e Shapley mostra que sempre existe um tal emparelhamento.

Podemos pensar no grafo bipartido como se fosse um conjunto de rapazes e um conjunto de moças. Ligamos um rapaz a uma moça por uma aresta se esse rapaz conhece essa moça (suponha que a relação de conhecimento seja simétrica). As funções preferência de que falamos acima podem ser interpretadas como a ordem de preferência que cada rapaz tem pelas moças que conhece e cada moça tem pelos rapazes que conhece. O fato de que as funções preferência são injetoras evita que ocorram empates na comparação de duas pessoas conhecidas. A prova do Teorema de Gale e Shapley que vamos 
apresentar a seguir é, fundamentalmente, a construção de um algoritmo que devolve um emparelhamento estável. O algoritmo segue as regras antigas da etiqueta: cada rapaz declara-se à moça de maior preferência e cada moça recusa todos os seus pretendentes exceto aquele que mais lhe agrada. Cada rapaz preterido torna a declarar-se à moça que segue na ordem de preferência, até que em algum momento, se não acha seu par, fica solteiro de vez.

Teorema 5 (Gale, Shapley): Para toda atribuição de preferências em um grafo bipartido existe um emparelhamento estável.

Prova. Seja $G$ um grafo $(A, B)$-bipartido. Suponha que $A$ seja o conjunto dos rapazes e $B$ o conjunto das moças. Seja $a^{+}$a moça preferida por $a \in A$ que ainda não o recusou. Seja $b^{+}$o rapaz que mais agrada a $b \in B$ e que já se declarou a $b$. Note que para qualquer vértice $v, v^{+}$muda ao longo do algoritmo. No começo temos um emparelhamento inicial $M_{0}=\emptyset$. Nas iterações ímpares $i(i=1,3, \ldots)$, cada rapaz $a$ declara-se à moça de maior preferência. Temos, então, um conjunto de arestas $F_{i}=\left\{a a^{+}: a \in A\right\}$ associado às declarações. Nas iterações pares $i+1$, cada moça $b$ recusa todos os pretendentes exceto $b^{+}$. Com isso, obtemos um emparelhamento $M_{i+1}=$ $\left\{b^{+} b \in F_{i}: b \in B\right\}$. As arestas de $F_{i}$ que não estão em $M_{i+1}$ representam encontros "mal-sucedidos" (para os moços). Para que o algoritmo não entre em loop, estas arestas não devem entrar novamente em nenhum $F_{j}, j>i$. De fato, isso não vai acontecer pois um rapaz recusado por uma moça jamais torna a declarar-se a ela (no nosso algoritmo). Cada rapaz declara-se a no máximo $|B|$ moças e portanto é recusado no máximo $|B|$ vezes. O algoritmo termina quando nenhuma moça recusar nenhum rapaz. Em cada iteração par, ocorre pelo menos uma recusa. E uma moça não recusa o mesmo rapaz duas vezes. Portanto é facil ver que o algoritmo termina após no máximo $2|A||B|$ iterações.

Para provar que o emparelhamento $M$ devolvido pelo algoritmo é estável, tome uma aresta $a b \in E(G) \backslash M$. Como $a b \notin M$, temos que $a$ nunca declarouse a $b$ ou foi recusado por $b$. Se $a$ nunca declarou-se a $b$ é porque casou-se $\operatorname{com} c$ e $\mu_{a}(c)>\mu_{a}(b)$. Se $a$ foi recusado por $b$ é porque $b$ casou-se com $d \mathrm{e}$ $\mu_{b}(d)>\mu_{b}(a)$. Portanto $M$ é estável.

Seja $G$ um grafo bipartido, e $\left\{\mu_{v}: v \in V(G)\right\}$ um conjunto de funções preferência. Dado um conjunto de arestas $F \subseteq E(G)$, definimos

$$
s_{F}(u v):=\left|\left\{u w \in F: \mu_{u}(w)>\mu_{u}(v)\right\}\right|+\left|\left\{w v \in F: \mu_{v}(w)>\mu_{v}(u)\right\}\right| .
$$


Observe que se $F, F^{\prime} \subseteq E(G)$ são conjuntos disjuntos, então $s_{F \cup F^{\prime}}=s_{F}+$ $s_{F^{\prime}}$. Ademais, se $F^{\prime} \subseteq F$, então $s_{F^{\prime}} \leq s_{F}$. Note também que, se $M$ é um emparelhamento estável, então $s_{M}(e) \geq 1$ para toda aresta $e \in E(G) \backslash M$.

Teorema 6: Se $G=(V, E)$ é um grafo bipartido e $\left\{\mu_{v}: v \in V\right\}$ é um conjunto de funções preferência para os vértices de $G$, então $L(G)$ é $\mathcal{L}$-colorível se $|\mathcal{L}(e)| \geq s_{E}(e)+1$ para toda aresta $e \in E$.

Prova. Vamos provar o teorema por indução no número de arestas do grafo. Se $E=\emptyset$, não há nada a fazer. Agora suponha $E \neq \emptyset$. Temos que provar que, para qualquer escolha das listas $\mathcal{L}(e)$, com $|\mathcal{L}(e)| \geq s_{E}(e)+1$ para todo $e \in E$, o grafo $L(G)$ é $\mathcal{L}$-colorível. Fixe arbitrariamente uma lista $\mathcal{L}(e)$, para cada aresta $e \in E$, cada uma delas com exatamente $s_{E}(e)+1$ cores. Seja $I$ o conjunto das arestas em cuja lista aparece uma certa cor $i$. Pelo Teorema de Gale e Shapley, o grafo $G[I]$ gerado pelas arestas em $I$ tem um emparelhamento estável $M$. Tome $G^{\prime}=G-M$. Ponha $E^{\prime}=E \backslash M$. Vamos provar que $G^{\prime}$ tem uma $\mathcal{L}^{\prime}$-coloração, onde $\mathcal{L}^{\prime}(e)=\mathcal{L}(e) \backslash\{i\}$ para todo $e \in E^{\prime}$. Para isso, basta provar que $\left|\mathcal{L}^{\prime}(e)\right| \geq s_{E^{\prime}}(e)+1$ para toda aresta $e \in E^{\prime}$ e aplicar a hipótese de indução. Note que, se $e \notin I$, então $\mathcal{L}^{\prime}(e)=\mathcal{L}(e) \geq s_{E}(e)+1 \geq s_{E^{\prime}}(e)+1$. O caso relevante é, então, $e \in I \backslash M$. Nesse caso,

$$
\left|\mathcal{L}^{\prime}(e)\right|=|\mathcal{L}(e)|-1 \geq s_{E}(e)=s_{E^{\prime} \cup M}(e)=s_{E^{\prime}}(e)+s_{M}(e) \geq s_{E^{\prime}}(e)+1
$$

para toda aresta $e \in E^{\prime}$. A última desigualdade é verdadeira porque $M$ é um emparelhamento estável. Por indução no número de arestas, $L\left(G^{\prime}\right)$ é $\mathcal{L}^{\prime}$-colorível. Podemos completar uma tal coloração pondo cor $i$ nas arestas de $M$. Desse modo, temos que $L(G)$ é $\mathcal{L}$-colorível.

Teorema 7 (Galvin): Se $G=(V, E)$ é um grafo bipartido, então $\operatorname{ch}^{\prime}(G)=$ $\chi^{\prime}(G)$.

Prova. Sejam $A$ e $B$ as partes de $G$. Fixe uma coloração $\varphi$ de $L(G)$ com $\chi^{\prime}(G)$ cores. Atribua preferências da seguinte maneira: um vértice $a \in A$ prefere um vizinho $b \in B$ a um vizinho $c \in B$ se $\varphi(a b)>\varphi(a c)$; um vértice $b \in B$ prefere um vizinho $a \in A$ a um vizinho $c \in A$ se $\varphi(b a)<\varphi(b c)$. Desse modo, $s_{E}(e) \leq \chi^{\prime}(G)-1$ para toda aresta $e \in E$. Assim, para qualquer escolha das listas $\mathcal{L}(e)$ com $|\mathcal{L}(e)| \geq \chi(L(G))$, o teorema anterior garante que $L(G)$ é $\mathcal{L}$-colorível. Portanto $\operatorname{ch}^{\prime}(G)=\chi^{\prime}(G)$. 


\subsection{Coloração total}

Um outro problema em coloração de grafos é o problema da coloração total. Uma coloração total de um grafo $G$ é uma atribuição de cores aos seus vértices e arestas, de modo que elementos adjacentes ou incidentes tenham cores diferentes. O número cromático total de um grafo $G$, denotado por $\chi_{T}(G)$, é o menor inteiro $k$ tal que o grafo $G$ pode ser colorido totalmente com $k$ cores. Um limitante fácil para o número cromático total é dado pelo algoritmo guloso de coloração de vértices e pelo teorema de Vizing: $2 \Delta+2$. No Capítulo 5, veremos limitantes bem melhores, porém todos eles ainda ficam longe do valor conjecturado.

Conjectura 2: Para todo grafo $G$, temos $\chi_{T}(G) \leq \Delta(G)+2$.

Note que se a Conjectura 1 for verdadeira, então esta conjectura é "quase" verdadeira: $\chi_{T}(G) \leq \Delta(G)+3$. Para ver isso pinte os vértices de um grafo usando $\Delta(G)+3$ cores. Para cada aresta teríamos uma lista de tamanho $\Delta(G)+3-2$ cores. Sob a hipótese de que Conjectura 1 é verdadeira, podemos colorir as arestas do grafo respeitando essas listas.

Recentemente B. Reed e M. Molloy [11] provaram que é possível colorir qualquer grafo totalmente com apenas $\Delta(G)+500$ cores. Este é o resultado mais próximo da conjectura que temos até o presente momento. 


\section{Capítulo 4}

\section{Ferramentas probabilísticas}

Este capítulo tem por única finalidade lembrar o leitor de algumas noções básicas de probabilidade e introduzir algumas ferramentas que serão usadas mais adiante. Poucos fatos serão demonstrados. Como referência básica de probabilidades, citamos [17].

\subsection{Conceitos básicos}

\section{Espaço de probabilidades}

Para nós, um espaço de probabilidade é um par ordenado $(\Omega, \mathbf{P r})$, onde $\Omega$ é um conjunto finito chamado espaço amostral e $\operatorname{Pr}: \Omega \longrightarrow[0,1]$ é uma função satisfazendo:

$$
\sum_{\omega \in \Omega} \operatorname{Pr}(\omega)=1
$$

Nos exemplos desta monografia, vamos considerar muitas vezes a distribuição uniforme de probabilidades que significa $\operatorname{Pr}(\omega)=1 /|\Omega|$ para todo $\omega \in \Omega$. Um evento num espaço de probabilidades é um subconjunto do seu espaço amostral. É usual estender a função $\operatorname{Pr}$ para todo evento $A \subseteq \Omega$ da seguinte maneira:

$$
\operatorname{Pr}(A)=\sum_{\omega \in A} \operatorname{Pr}(\omega)
$$

Uma propriedade que vamos usar muito é a cota da união:

$$
\operatorname{Pr}\left(\bigcup_{i=1}^{m} A_{i}\right) \leq \sum_{i=1}^{m} \operatorname{Pr}\left(A_{i}\right) .
$$




\section{Probabilidade condicional}

Vamos definir probabilidade condicional de $A$ dado $B$, quando $\operatorname{Pr}(B)>0$, como

$$
\operatorname{Pr}(A \mid B)=\frac{\operatorname{Pr}(A \cap B)}{\operatorname{Pr}(B)} .
$$

Usando apenas a definição de propriedade condicional, somos capazes de escrever $\operatorname{Pr}\left(A_{1} \cap \cdots \cap A_{n}\right)$ em uma forma que nos será especialmente útil na demonstração do Lema Local:

$$
\begin{aligned}
& \operatorname{Pr}\left(\bigcap_{i=1}^{n} A_{i}\right) \\
= & \operatorname{Pr}\left(A_{1} \mid \bigcap_{i=2}^{n} A_{i}\right) \times \operatorname{Pr}\left(\bigcap_{i=2}^{n} A_{i}\right) \\
= & \operatorname{Pr}\left(A_{1} \mid \bigcap_{i=2}^{n} A_{i}\right) \times \operatorname{Pr}\left(A_{2} \mid \bigcap_{i=3}^{n} A_{i}\right) \times \operatorname{Pr}\left(\bigcap_{i=3}^{n} A_{i}\right) \\
\vdots & \prod_{i \in[n]} \operatorname{Pr}\left(A_{i} \mid \bigcap_{\substack{j \in[n] \\
j>i}} A_{j}\right) .
\end{aligned}
$$

Como a intersecção é uma operação comutativa, podemos "descascar" a probabilidade acima em qualquer ordem. Portanto, para qualquer permutação $\pi$ de $n$ elementos, vale que

$$
\operatorname{Pr}\left(\bigcap_{i=1}^{n} A_{i}\right)=\prod_{i \in[n]} \operatorname{Pr}\left(A_{\pi(i)} \mid \bigcap_{\substack{j \in[n] \\ j>i}} A_{\pi(j)}\right) .
$$

\section{Independência mútua}

Um evento $A$ é mutuamente independente de um conjunto de outros eventos $\mathcal{E}$ se para todo $\mathcal{F} \subseteq \mathcal{E}$, temos

$$
\operatorname{Pr}\left(A \mid \bigcap_{B \in \mathcal{F}} B\right)=\operatorname{Pr}(A),
$$

isto é, a probabilidade condicional de $A$ dado que ocorreram todos os eventos em $\mathcal{F}$ é igual à probabilidade de ocorrer $A$. 
Um fato simples, que vamos apenas mencionar, é o seguinte. Se $A$ é mutuamente independente de um conjunto de eventos $\mathcal{E}$, e $\mathcal{I}, \mathcal{F} \subseteq \mathcal{E}$, então

$$
\operatorname{Pr}\left(A \mid \bigcap_{B \in \mathcal{F}} B \cap \bigcap_{C \in \mathcal{I}} \bar{C}\right)=\operatorname{Pr}(A) .
$$

\section{Variável aleatória}

Uma variável aleatória $X$ sobre um espaço de probabilidade $(\Omega, \mathbf{P r})$ é uma função $X: \Omega \longrightarrow \mathbb{R}$. Seja $\Omega_{X}=X(\Omega)$ o conjunto de valores que a função $X$ pode assumir. A variável aleatória $X$ define um espaço de probabilidades $\left(\Omega_{X}, \mathbf{P r}_{X}\right)$ onde, para cada $x \in \Omega_{X}$,

$$
\operatorname{Pr}_{X}(x)=\sum_{\substack{\omega \in \Omega \\ X(\omega)=x}} \operatorname{Pr}(\omega) .
$$

Quando a variável aleatória em questão estiver bem determinada, é comum abusarmos da notação e escrevermos $\operatorname{Pr}(x)$ em vez de $\operatorname{Pr}_{X}(x)$. Às vezes, denotamos um evento implicitamente, por exemplo, escrevendo $X \geq x_{0}$ para denotar o evento $\left\{x \in \Omega_{X}: x \geq x_{0}\right\}$.

\section{Valor esperado}

A esperança da variável aleatória $X$, denotada por $\mathbf{E}(X)$, é uma média ponderada dos valores assumidos por $X$ :

$$
\mathbf{E}(X)=\sum_{\omega \in \Omega} \operatorname{Pr}(\omega) X(\omega)=\sum_{x \in \Omega_{X}} x \operatorname{Pr}(x) .
$$

A propriedade da linearidade da esperança também será bastante empregada ao longo do nosso texto:

$$
\mathbf{E}\left(\sum_{i=1}^{m} X_{i}\right)=\sum_{i=1}^{m} \mathbf{E}\left(X_{i}\right) .
$$

\subsection{Método do Primeiro Momento}

O Princípio do Primeiro Momento resume-se no seguinte fato: se a média de $n$ valores reais $v_{1}, \ldots, v_{n}$ é $v$, então existe um índice $i$ tal que $v_{i} \geq v$. Equivalentemente, se $\mathbf{E}(X) \geq t$, então $\operatorname{Pr}(X \geq t)>0$. Ou, por simetria, se $\mathbf{E}(X) \leq t$, então $\operatorname{Pr}(X \leq t)>0$. 


\section{Desigualdade de Markov}

Suponha que $X$ é uma variável aleatória não-negativa $(X(\omega) \geq 0$ para todo $\omega \in \Omega$ ). A Desigualdade de Markov limita, para todo $t>0$, a probabilidade de que $X$ assuma um valor muito acima de sua média:

$$
\operatorname{Pr}(X \geq t) \leq \frac{\mathbf{E}(X)}{t} .
$$

A prova desse fato é muito simples. Basta escrever $\mathbf{E}(X)$ como

$$
\begin{aligned}
\mathbf{E}(X) & =\sum_{\substack{x \in \Omega_{X}\\
}} x \operatorname{Pr}(x) \\
& =\sum_{\substack{x \in \Omega_{X} \\
x<t}} x \operatorname{Pr}(x)+\sum_{\substack{x \in \Omega_{X} \\
x \geq t}} x \operatorname{Pr}(x) \\
& \geq \sum_{\substack{x \in \Omega_{X} \\
x \geq t}} t \operatorname{Pr}(x) \\
& =t \operatorname{Pr}(X \geq t)
\end{aligned}
$$

A desigualdade segue.

Neste texto vamos aplicar a Desigualdade de Markov quando $X$ é uma variável aleatória que assume valores inteiros. Especificamente, vamos usar o seguinte fato que decorre da Desigualdade de Markov quando $X$ é inteira e não-negativa:

$$
\operatorname{Pr}(X>0) \leq \mathbf{E}(X) .
$$

\subsection{O Lema Local}

Uma outra ferramenta útil é o Lema Local. Suponha que temos um conjunto $\mathcal{E}$ de eventos "ruins" que queremos evitar, e que cada evento $A \in \mathcal{E}$ é mutuamente independente de todos os outros eventos em $\mathcal{E}$ exceto um número limitado $d$ deles. Ou seja, para todo $A \in \mathcal{E}$, existe um conjunto $\mathcal{F}_{A} \subseteq \mathcal{E}$ tal que $A$ é mutuamente independente de $\mathcal{F}_{A}$ e $\left|\mathcal{F}_{A}\right| \geq|\mathcal{E}|-d$. Se $\operatorname{Pr}(A) \leq p$ para todo $A \in \mathcal{E}$, e se $4 p d \leq 1$, então com probabilidade positiva nenhum dos eventos em $\mathcal{E}$ ocorre. Isto é, existe $\omega \in \Omega$ tal que

$$
\omega \notin \bigcup_{A \in \mathcal{E}} A \quad \text { e } \quad \operatorname{Pr}(\omega)>0 .
$$




\section{Demonstração do Lema Local}

Assim como em [2], vamos primeiramente provar uma versão assimétrica do Lema Local.

Lema 8 (Lema Local): Seja $\mathcal{E}=\left\{A_{1}, \ldots, A_{n}\right\}$ um conjunto de eventos em um espaço de probabilidades qualquer. Suponha que, para todo $1 \leq i \leq n$, tenhamos um conjunto $J_{i} \subseteq[n]$ tal que o evento $A_{i}$ é mutuamente independente dos eventos no conjunto $\left\{A_{j}: j \in J_{i}\right\}$. Se existem $x_{1}, \ldots, x_{n} \in[0,1)$ tais que

$$
\operatorname{Pr}\left(A_{i}\right) \leq x_{i} \times \prod_{j \in[n] \backslash J_{i}}\left(1-x_{j}\right)
$$

para todo $i$, então

$$
\operatorname{Pr}\left(\bigcap_{i=1}^{n} \bar{A}_{i}\right) \geq \prod_{i=1}^{n}\left(1-x_{i}\right) .
$$

Prova. Vamos provar que, para todo $J \subsetneq[n]$, e para todo $i \notin J$ temos

$$
\operatorname{Pr}\left(A_{i} \mid \bigcap_{j \in J} \bar{A}_{j}\right) \leq x_{i}
$$

A prova desse fato é por indução em $|J|$. Se $J=\emptyset$, então a afirmação é verdadeira pois certamente $\operatorname{Pr}\left(A_{i}\right) \leq x_{i}$. Suponha que $|J|>0$ e suponha que a afirmação seja válida para conjuntos com cardinalidade menor que $|J|$. Observe que

$$
\begin{aligned}
\operatorname{Pr}\left(A_{i} \mid \bigcap_{j \in J} \bar{A}_{j}\right) & =\operatorname{Pr}\left(A_{i} \mid \bigcap_{j \in J \backslash J_{i}} \bar{A}_{j} \cap \bigcap_{j \in J_{i} \cap J} \bar{A}_{j}\right) \\
& =\operatorname{Pr}\left(A_{i} \cap \bigcap_{j \in J \backslash J_{i}} \bar{A}_{j} \mid \bigcap_{j \in J_{i} \cap J} \bar{A}_{j}\right) / \operatorname{Pr}\left(\bigcap_{j \in J \backslash J_{i}} \bar{A}_{j} \mid \bigcap_{j \in J_{i} \cap J} \bar{A}_{j}\right) \\
& \leq \operatorname{Pr}\left(A_{i} \mid \bigcap_{j \in J_{i} \cap J} \bar{A}_{j}\right) / \operatorname{Pr}\left(\bigcap_{j \in J \backslash J_{i}} \bar{A}_{j} \mid \bigcap_{j \in J_{i} \cap J} \bar{A}_{j}\right) \\
& \leq \operatorname{Pr}\left(A_{i}\right) / \operatorname{Pr}\left(\bigcap_{j \in J \backslash J_{i}} \bar{A}_{j} \mid \bigcap_{j \in J_{i} \cap J} \bar{A}_{j}\right) .
\end{aligned}
$$


Note que, usando a propriedade de probabilidades condicionais que mencionamos, temos

$$
\begin{aligned}
\operatorname{Pr}\left(\bigcap_{j \in J \backslash J_{i}} \bar{A}_{j} \mid \bigcap_{j \in J_{i} \cap J} \bar{A}_{j}\right) & =\prod_{j^{\prime} \in J \backslash J_{i}} \operatorname{Pr}\left(\bar{A}_{j^{\prime}} \mid \bigcap_{\substack{k \in J \backslash J_{i} \\
k<j^{\prime}}} \bar{A}_{k} \cap \bigcap_{j \in J_{i} \cap J} \bar{A}_{j}\right) \\
& =\prod_{\substack { j^{\prime} \in J \backslash J_{i} \\
\begin{subarray}{c}{k \in J \backslash J_{i} \\
k<j^{\prime}{ j ^ { \prime } \in J \backslash J _ { i } \\
\begin{subarray} { c } { k \in J \backslash J _ { i } \\
k < j ^ { \prime } } }\end{subarray}}\left(1-\operatorname{Pr}\left(A_{j^{\prime}} \mid \bigcap_{k} \bigcap_{j \in J_{i} \cap J} \bar{A}_{j}\right)\right) .
\end{aligned}
$$

Por hipótese de indução, sabemos que

$$
\prod_{j^{\prime} \in J \backslash J_{i}}\left(1-\operatorname{Pr}\left(A_{j^{\prime}} \mid \bigcap_{\substack{k \in J \backslash J_{i} \\ k<j^{\prime}}} \bar{A}_{k} \cap \bigcap_{j \in J_{i} \cap J} \bar{A}_{j}\right)\right) \geq \prod_{j \in J \backslash J_{i}}\left(1-x_{j}\right) .
$$

Assim,

$$
\begin{aligned}
\operatorname{Pr}\left(A_{i} \mid \bigcap_{j \in J} \bar{A}_{j}\right) & \leq \operatorname{Pr}\left(A_{i}\right) / \operatorname{Pr}\left(\bigcap_{j \in J \backslash J_{i}} \bar{A}_{j} \mid \bigcap_{j \in J_{i} \cap J} \bar{A}_{j}\right) \\
& \leq x_{i} \times \prod_{j \in[n] \backslash J_{i}}\left(1-x_{j}\right) / \prod_{j \in J \backslash J_{i}}\left(1-x_{j}\right) \\
& \leq x_{i} .
\end{aligned}
$$

Agora, provar a asserção do Lema, basta escrever a probabilidade em que estamos interessados na forma adequada.

$$
\begin{aligned}
\operatorname{Pr}\left(\bigcap_{i=1}^{n} \bar{A}_{i}\right) & =\prod_{i \in[n]} \operatorname{Pr}\left(\bar{A}_{i} \mid \bigcap_{\substack{k \in[n] \\
k<i}} \bar{A}_{k}\right) \\
& =\prod_{i \in[n]}\left(1-\operatorname{Pr}\left(A_{i} \mid \bigcap_{\substack{k \in[n] \\
k<i}} \bar{A}_{k}\right)\right) \\
& \geq \prod_{i \in[n]}\left(1-x_{i}\right) .
\end{aligned}
$$

Corolário 9 (Lema Local; Versão simétrica): Suponha que temos um conjunto $\mathcal{E}=\left\{A_{1}, \ldots, A_{n}\right\}$ de eventos num espaço de probabilidades qualquer. 
Suponha que, para cada evento $A_{i} \in \mathcal{E}$, exista um conjunto de indices $J_{i}$ tal que $A_{i}$ é mutuamente independente dos eventos em $\left\{A_{j}: j \in J_{i}\right\}$. Se $\left|J_{i}\right| \geq|\mathcal{E}|-d$ e $\operatorname{Pr}\left(A_{i}\right) \leq p$, para todo $i \in[n]$, e se $4 p d \leq 1$, então com probabilidade positiva nenhum dos eventos em $\mathcal{E}$ ocorre, isto é,

$$
\operatorname{Pr}\left(\bigcap_{i=1}^{n} \overline{A_{i}}\right)>0 .
$$

Prova. Ponha $x_{i}=2 \operatorname{Pr}\left(A_{i}\right) \leq 2 p$. Como $4 p d \leq 1, x_{i} \leq \frac{1}{2 d}$. Se $c=2 \ln 2$, então $(1-x) \geq \mathrm{e}^{-c x}$, quando $x \leq \frac{1}{2}$. Note que $d \geq 1$. Podemos então dizer que

$$
\begin{aligned}
x_{i} \times \prod_{j \in[n] \backslash J_{i}}\left(1-x_{j}\right) & \geq x_{i} \times \prod_{j \in[n] \backslash J_{i}} \mathrm{e}^{-c x_{i}} \\
& =2 \operatorname{Pr}\left(A_{i}\right) \times \exp \left\{-c \sum_{j \in[n] \backslash J_{i}} 2 \operatorname{Pr}\left(A_{i}\right)\right\} \\
& \geq 2 \operatorname{Pr}\left(A_{i}\right) \times \mathrm{e}^{-c / 2} \\
& =\operatorname{Pr}\left(A_{i}\right) .
\end{aligned}
$$

Agora, aplicando o Lema 8, segue diretamente que

$$
\operatorname{Pr}\left(\bigcap_{i=1}^{n} \overline{A_{i}}\right)>0 .
$$

A prova do corolário está completa.

\subsection{Ferramentas de concentração}

Muitas vezes é útil sabermos que com alta probabilidade uma variável assume valores próximos da sua média. Aqui vamos listar algumas desigualdades que servem a esse propósito.

\section{Variância}

A variância de uma variável aleatória $X$ é uma espécie de medida de concentração de $X$. Ela é definida por

$$
\operatorname{Var}(X)=\mathbf{E}\left((X-\mathbf{E}(X))^{2}\right)
$$


Note que $\operatorname{Var}(X)=0$ se e somente se a variável $X$ não tem absolutamente nada de aleatória, isto é, se e somente se $X$ assume um certo valor com probabilidade 1 .

\section{A Desigualdade de Chebyshev}

Suponha que $\sigma^{2}=\operatorname{Var}(X)=\mathbf{E}\left((X-\mathbf{E}(X))^{2}\right)$. A Desigualdade de Chebyshev afirma que para todo $\lambda>0$,

$$
\operatorname{Pr}(|X-\mathbf{E}(X)| \geq \lambda \sigma) \leq \frac{1}{\lambda^{2}} .
$$

Para provar a desigualdade de Chebyshev podemos usar a desigualdade de Markov:

$$
\begin{aligned}
\operatorname{Pr}(|X-\mathbf{E}(X)| \geq \lambda \sigma) & =\operatorname{Pr}\left((X-\mathbf{E}(X))^{2} \geq(\lambda \sigma)^{2}\right) \\
& \leq \frac{\mathbf{E}\left((X-\mathbf{E}(X))^{2}\right)}{(\lambda \sigma)^{2}} \\
& =\frac{\sigma^{2}}{(\lambda \sigma)^{2}} \\
& =\frac{1}{\lambda^{2}}
\end{aligned}
$$

\section{O Limitante de Chernoff}

Se $X$ é soma de $n$ variáveis aleatórias que assumem valores em $\{0,1\}$ independentemente, cada qual é 1 com probabilidade $p$ e 0 com probabilidade $1-p$, então dizemos que $X$ possui distribuição binomial com parâmetros $n$ e $p$. O Limitante de Chernoff nos dá uma medida de quão concentrada $X$ está em torno de sua média $n p$. Para $0 \leq t \leq n p$, tem-se

$$
\operatorname{Pr}(|X-n p|>t)<2 e^{-t^{2} / 3 n p} .
$$

O Limitante de Chernoff so é interessante quando $t \geq \sqrt{3 n p \ln 2}$. Vamos nos referir genericamente a uma variável binomial com parâmetros $n$ e $p$ escrevendo $\mathbf{B I N}(n, p)$.

\section{Um limitante para binomiais}

Vamos usar no Capítulo 6 o seguinte fato: 
Fato 1: Para qualquer constante positiva $c$, para todo inteiro $n \geq c$, e para qualquer inteiro positivo $k$,

$$
\operatorname{Pr}\left(\operatorname{BIN}\left(n, \frac{c}{n}\right) \geq k\right) \leq \frac{c^{k}}{k !} .
$$

Prova. Se $n<k$ não há nada a fazer. Suponha que $n \geq k$. Para provar a desigualdade, sejam $X_{1}, \ldots, X_{n}$ variáveis aleatórias independentes que assumem valores em $\{0,1\}$, cada qual com probabilidade $c / n$ de valer 1 e $1-c / n$ de valer 0. Por definição, $\operatorname{BIN}(n, c / n)=X_{1}+\cdots+X_{n}$. Considere o evento $E$ como sendo $X_{1}+\cdots+X_{n} \geq k$. Para limitar a probabilidade de $E$, vamos considerar eventos $E_{I}, I \in\left(\begin{array}{c}{[n]} \\ k\end{array}\right)$. Dizemos que um evento $E_{I}$ acontece quando

$$
\sum_{i \in I} X_{i}=k
$$

Note que $\operatorname{Pr}\left(E_{I}\right) \leq c^{k} / n^{k}$ para todo $I \in\left(\begin{array}{c}{[n]} \\ k\end{array}\right)$. Pela cota da união, temos

$$
\operatorname{Pr}(E)=\operatorname{Pr}\left(\bigcup_{I \in\left(\begin{array}{c}
{[n]} \\
k
\end{array}\right)} E_{I}\right) \leq \sum_{I \in\left(\begin{array}{c}
{[n]} \\
k
\end{array}\right)} \operatorname{Pr}\left(E_{I}\right) \leq\left(\begin{array}{l}
n \\
k
\end{array}\right) \times \frac{c^{k}}{n^{k}} \leq \frac{n^{k}}{k !} \times \frac{c^{k}}{n^{k}}=\frac{c^{k}}{k !}
$$

\section{A Desigualdade de Talagrand}

Uma outra desigualdade bem conhecida, que será empregada mais adiante, é a Desigualdade de Talagrand. A versão original dessa desigualdade mostra que, sob certas condições, uma variável aleatória está concentrada em torno de sua mediana. Na aplicação que faremos da Desigualdade de Talagrand, vamos usar uma outra versão, que foi retirada de [12]. Esta, decorrente da primeira, mostra uma concentração em torno da média e não da mediana.

Desigualdade de Talagrand: Seja $X$ uma variável aleatória não-negativa $e$ não identicamente nula, determinada por $n$ experimentos independentes $T_{1}, \ldots, T_{n}$. Suponha que existam $c, r>0$ tais que:

i. Mudando a saída de um único experimento, o valor de $X$ é afetado de no máximo c;

ii. Para qualquer $s$, se $X \geq s$, então existem no máximo rs experimentos cujas saídas certificam, de alguma maneira, que $X \geq s$. 
Então para qualquer $0 \leq t \leq \mathbf{E}(X)$, temos

$$
\operatorname{Pr}(|X-\mathbf{E}(X)|>t+60 c \sqrt{r \mathbf{E}(X)}) \leq 4 \exp \left\{-t^{2} / 8 c^{2} r \mathbf{E}(X)\right\} .
$$

Na Seção 6.3 do Capítulo 6, vamos aplicar a Desigualdade de Talagrand fazendo as contas com um certo nível de detalhes. Das próximas vezes, vamos simplesmente dizer que $\operatorname{Pr}(|X-\mathbf{E}(X)|>t) \leq 4 e^{-\beta t^{2} / \mathbf{E}(X)}$, onde $\beta$ é uma constante apropriada, que dá conta do termo $60 c \sqrt{r \mathbf{E}(X)}$ ignorado, e que geralmente não atrapalha na obtenção da cota desejada. 


\section{Capítulo 5}

\section{Resultados probabilísticos em coloração}

\subsection{Número lista cromático}

Já vimos que o número lista-cromático não está relacionado com o número cromático. A seguir, vamos apresentar um teorema de Alon que mostra que o número lista-cromático está relacionado com o número de coloração, isto é, se um deles cresce, então o outro também cresce, ainda que em "velocidades" diferentes. A demonstração apresentada aqui foi encontrada em [1] e difere ligeiramente da contida na bibliografia estudada [12].

O método da coloração gulosa nos dá um lado da história: $\operatorname{ch}(G) \leq$ $\hat{\delta}(G)+1$. O outro lado é dado pelo Teorema de Alon. Claramente, todo grafo $G$ possui um subgrafo $H$ com grau médio $d$ pelo menos $\hat{\delta}(G)$. Para um tal subgrafo, o Teorema de Alon afirma que

$$
d \leq 4\left(\begin{array}{c}
\operatorname{ch}(H)^{4} \\
\operatorname{ch}(H)
\end{array}\right) \ln 2\left(\begin{array}{c}
\operatorname{ch}(H)^{4} \\
\operatorname{ch}(H)
\end{array}\right) .
$$

Como $\operatorname{ch}(H) \leq \operatorname{ch}(G)$, temos que

$$
\hat{\delta}(G) \leq d \leq 4\left(\begin{array}{c}
\operatorname{ch}(G)^{4} \\
\operatorname{ch}(G)
\end{array}\right) \ln 2\left(\begin{array}{c}
\operatorname{ch}(G)^{4} \\
\operatorname{ch}(G)
\end{array}\right) .
$$

Fazendo as contas (que vamos omitir), podemos obter que

$$
c h=\Omega(\ln \hat{\delta}(G) / \ln \ln \hat{\delta}(G)) .
$$


Lema 10: Se $G$ é um grafo com grau médio pelo menos d, então existe um subgrafo bipartido $H \subseteq G$ tal que $\delta(H)>d / 4$.

Prova. Primeiramente, podemos eliminar, recursivamente, os vértices de $G$ que possuem grau menor ou igual a $d / 2$. Note que, ao eliminarmos um vértice $v$ desse tipo, o novo grau médio $d^{\prime}$ não diminui:

$$
d^{\prime}=\frac{\sum_{u \in V(G)} d(u)-2 d(v)}{n-1} \geq \frac{n d-2 d(v)}{n-1} \geq \frac{n d-d}{n-1}=d .
$$

Chame $G^{\prime}$ ao grafo resultante. Agora tomamos $H \subseteq G^{\prime}$ como sendo um subgrafo bipartido gerador com o maior número de arestas possível. O subgrafo $H$ possui grau mínimo $\delta(H)>d / 4$, caso contrário, seja $v \in H$ um vértice com grau menor que $d / 4$ (em $H$ ). Como $v \in V\left(G^{\prime}\right)$ e $\delta\left(G^{\prime}\right)>d / 2$, temos que, passando $v$ para a outra parte de $H$, aumentamos o número de arestas de $H$ : absurdo. Assim, $H \subseteq G^{\prime} \subseteq G$ é um subgrafo bipartido de $G$ com grau mínimo maior que $d / 4$.

Teorema 11 (Alon): Seja G um grafo com grau médio pelo menos d. Se s é um inteiro positivo e

$$
d>4\left(\begin{array}{c}
s^{4} \\
s
\end{array}\right) \ln 2\left(\begin{array}{c}
s^{4} \\
s
\end{array}\right)
$$

então $\operatorname{ch}(G)>s$.

Prova. Seja $H \subseteq G$ um subgrafo $(A, B)$-bipartido com grau mínimo pelo menos $d / 4$ como garante o Lema 10 . Suponha, sem perda de generalidade, que $|A| \geq|B|$. Vamos atribuir listas de cores $\mathcal{L}(v)$ a cada vértice $v \in V(H)$, cada uma delas com tamanho $s$. Se conseguirmos fazer isso de modo que $H$ não seja $\mathcal{L}$-colorível, então teremos provado que $\operatorname{ch}(H)>s$. Note que isso é suficiente para provar o teorema pois

$$
\operatorname{ch}(G) \geq \operatorname{ch}(H)
$$

As cores serão tomadas do conjunto $\left[s^{4}\right]$. Primeiramente, vamos sortear uma $s$-lista $\mathcal{L}(b)$ uniformemente e independentemente para cada vértice $b \in B$. Ou seja, nosso espaço de probabilidades é $(\Omega, \mathbf{P r})$, onde

$$
\Omega=\left(\begin{array}{c}
{\left[s^{4}\right]} \\
s
\end{array}\right)^{|B|} \text { e } \quad \operatorname{Pr}(\omega)=\left(\begin{array}{c}
s^{4} \\
s
\end{array}\right)^{-|B|}
$$


para todo $\omega \in \Omega$. Chamamos um vértice $a \in A$ de ruim, se

$$
\{\mathcal{L}(b): b \in \Gamma(a)\}=\left(\begin{array}{c}
{\left[s^{4}\right]} \\
s
\end{array}\right),
$$

isto é, se cada um dos possíveis $s$-subconjuntos de $\left[s^{4}\right]$ aparecer como lista de algum vizinho de $a$. Seja $X_{a}$ a variável aleatória

$$
X_{a}= \begin{cases}1 & \text { se } a \text { é ruim, } \\ 0 & \text { caso contrário. }\end{cases}
$$

Podemos estimar a probabilidade de $X_{a}=1$ :

$$
\begin{aligned}
\operatorname{Pr}\left(X_{a}=1\right) & =1-\operatorname{Pr}\left(X_{a}=0\right) \\
& \geq 1-\sum_{W \subseteq\left[s^{4}\right],|W|=s} \operatorname{Pr}(W \neq \mathcal{L}(b) \forall b \in \Gamma(a)) \\
& =1-\left(\begin{array}{c}
s^{4} \\
s
\end{array}\right)\left(1-\left(\begin{array}{c}
s^{4} \\
s
\end{array}\right)^{-1}\right)^{d_{H}(a)} .
\end{aligned}
$$

Vamos usar agora a desigualdade bem conhecida $(1-x)<e^{-x}, x \neq 0$. Como $d_{H}(a) \geq d / 4$, podemos escrever:

$$
\begin{aligned}
\operatorname{Pr}\left(X_{a}=1\right) & >1-\left(\begin{array}{c}
s^{4} \\
s
\end{array}\right) \exp \left\{-\left(\begin{array}{c}
s^{4} \\
s
\end{array}\right)^{-1} \frac{d}{4}\right\} \\
& >1-\left(\begin{array}{c}
s^{4} \\
s
\end{array}\right) \exp \left\{-\left(\begin{array}{c}
s^{4} \\
s
\end{array}\right)^{-1}\left(\begin{array}{c}
s^{4} \\
s
\end{array}\right) \ln 2\left(\begin{array}{c}
s^{4} \\
s
\end{array}\right)\right\} \\
& =1-1 / 2 \\
& =1 / 2 .
\end{aligned}
$$

Assim, a probabilidade de um vértice $a \in A$ ser ruim é maior que $1 / 2$. Seja $X$ a variável aleatória que representa o número de vértices ruins em $A$. É claro que $X=\sum_{a \in A} X_{a}$. Pela linearidade da esperança temos:

$$
\mathbf{E}(X)=\mathbf{E}\left(\sum_{a \in A} X_{a}\right)=\sum_{a \in A} \mathbf{E}\left(X_{a}\right)>|A| / 2 .
$$

Então, a esperança do número de vértices ruins em $A$ é maior que $|A| / 2$. Pelo princípio do primeiro momento, podemos afirmar que existe uma atribuição de listas aos vértices em $B$ tal que existem mais que $|A| / 2$ vértices ruins em $A$. Fixe uma tal atribuição parcial de listas e chame-a $\mathcal{L}_{B}$. 
O que vamos fazer agora é achar um conjunto de listas $\mathcal{L}_{A}$ para os vértices de $A$ de forma que qualquer $\left(\mathcal{L}_{A} \cup \mathcal{L}_{B}\right)$-coloração de $H$ não é própria. Sorteie listas $\mathcal{L}(a)$ independentemente e uniformemente para os vértices de $A$, exatamente como fizemos para a parte $B$. Fixe arbitrariamente uma $\mathcal{L}_{B}$-coloração $\varphi_{B}$ dos vértices em $B$. Seja $a \in A$ um vértice ruim. Por definição, qualquer conjunto contendo $s$ cores é a lista de algum vizinho $b$ de $a$. Pela coloração $\varphi_{B}$ fixada, $b$ recebe alguma dessas $s$ cores. Então, para cada vértice ruim $a \in A$, no máximo $s-1$ cores podem ser colocadas em $a$ sem que entrem em conflito com as cores dadas por $\varphi_{B}$ aos seus vizinhos.

Assim, para que possamos $\mathcal{L}$-colorir $H$, é preciso que a lista de cada vértice ruim contenha pelo menos uma cor "compatível" com $\varphi_{B}$. Seja a variável aleatória:

$$
Y_{a}= \begin{cases}1 & \text { se } \mathcal{L}_{A}(a) \text { tem uma cor "compatível" com } \varphi_{B} \\ 0 & \text { caso contrário. }\end{cases}
$$

Podemos delimitar $\operatorname{Pr}\left(Y_{a}=1\right)$ da seguinte forma:

$$
\operatorname{Pr}\left(Y_{a}=1\right) \leq \frac{(s-1)\left(\begin{array}{c}
s^{4}-1 \\
s-1
\end{array}\right)}{\left(\begin{array}{c}
s^{4} \\
s
\end{array}\right)}=\frac{s(s-1)}{s^{4}}<1 / s^{2} .
$$

A estimativa acima é bem grosseira, mas é suficiente para a análise que queremos. Como temos $k>|A| / 2$ vértices ruins em $A$, a probabilidade de que todos eles tenham uma cor "compatível" em suas listas é menor que

$$
\left(1 / s^{2}\right)^{k}<\left(1 / s^{2}\right)^{|A| / 2}=1 / s^{|A|} \leq 1 / s^{|B|},
$$

e portanto, a probabilidade de que possamos estender $\varphi_{B}$ a uma $\mathcal{L}$-coloração de $H$ é menor que $1 / s^{|B|}$. Mas temos $s^{|B|}$ maneiras de escolher a $\mathcal{L}_{B}$-coloração $\varphi_{B}$. Assim, a probabilidade de que $H$ seja $\mathcal{L}$-colorível é menor que

$$
\frac{1}{s^{|B|}} s^{|B|}=1
$$

Se a probabilidade de $H$ ser $\mathcal{L}$-colorível é menor que 1 , então existe um modo de escolher as listas para a parte $A$ de modo que $H$ não seja $\mathcal{L}$-colorível. Fixe um tal conjunto de listas e dê nome $\mathcal{L}_{A}$. Ponha $\mathcal{L}=\mathcal{L}_{A} \cup \mathcal{L}_{B}$. Acabamos de mostrar que $H$ não é $\mathcal{L}$-colorível, portanto $\operatorname{ch}(H)>s$ e o teorema está provado. 
A conjectura a seguir foi proposta por B. Reed [14] e envolve apenas a estrutura de intersecção das listas associadas aos vértices, e não o tamanho das listas. Esta conjectura foi desprovada por Bohman e Holzman em [3].

Conjectura 3 (Falsa): Seja $G$ um grafo e, para cada $v \in V(G)$, seja $\mathcal{L}(v)$ uma lista com pelo menos $\ell$ cores. Se, para todo $v$, cada cor da lista $\mathcal{L}(v)$ está na lista de no máximo $\ell-1$ vizinhos de $v$, então $G$ é $\mathcal{L}$-colorível.

A seguir temos um teorema que é uma versão fraca da conjectura.

Teorema 12 (Reed [14]): Seja $G$ um grafo e, para cada vértice $v \in V(G)$, seja $\mathcal{L}(v)$ uma lista com pelo menos $\ell$ cores. Se cada cor em $\mathcal{L}(v)$ aparece na lista de no máximo $\ell / 8$ vizinhos de $v$, então $G$ é $\mathcal{L}$-colorivel.

Prova. Podemos truncar as listas para que possuam todas tamanho $\ell$. A seguir, sorteamos para cada vértice uma cor de sua lista. Suponha que a escolha é uniforme e independente para cada vértice do grafo. O que nos incomoda é que podemos sortear, por azar, a mesma cor para ambas as pontas de uma mesma aresta. Temos que evitar, então, todos os eventos ruins da forma $A_{e, i}$, que significam dar a cor $i$ a ambas as pontas de $e$. Queremos evitar que ocorram os eventos do conjunto $\mathcal{E}=\left\{A_{u} v, i\right.$ : uv $\in$ $E(G), i \in \mathcal{L}(u) \cap \mathcal{L}(v)\}$.

A fim de aplicarmos o Lema Local, devemos fazer duas coisas. Primeiramente, devemos achar um limitante adequado $p$ para a probabilidade dos eventos em $\mathcal{E}$. Devemos também mostrar que cada evento em $\mathcal{E}$ é mutuamente independente de todos os outros eventos em $\mathcal{E}$ exceto no máximo $d$ desses eventos, para algum $d$ tal que $4 p d \geq 1$.

A probabilidade de cada evento em $\mathcal{E}$ é $p=1 / \ell^{2}$. Seja $e=u v \in E(G)$ uma aresta arbitrária. Observe que a ocorrencia de um determinado evento $A_{e, i}$ depende somente das cores atribuídas às pontas de $e$. Portanto, o evento $A_{e, i}$ é mutuamente independente dos eventos em $\mathcal{E} \backslash S_{e}$, onde $S_{e}=\left\{A_{f, j}: j \in\right.$ $\mathcal{L}(u), u$ é ponta de $f\} \cup\left\{A_{f, j}: j \in \mathcal{L}(v), v\right.$ é ponta de $\left.f\right\}$. Como $\mathcal{L}(u)$ (respectivamente $\mathcal{L}(v)$ ) tem apenas $\ell$ cores, e como cada uma delas aparece na lista de no máximo $\ell / 8$ vizinhos de $u$ (respectivamente $v$ ), temos que $\left|S_{e}\right| \leq \ell^{2} / 8+\ell^{2} / 8=\ell^{2} / 4$. Pela arbitráredade com que tomamos $e$, podemos afirmar que qualquer evento em $\mathcal{E}$ é mutuamente independente de todos os outros eventos em $\mathcal{E}$ exceto no máximo $d=\ell^{2} / 4$ desses eventos. Fazendo as 
contas,

$$
4 p d \leq 4 \times \frac{1}{\ell^{2}} \times \frac{\ell^{2}}{4} \leq 1 .
$$

Pelo Lema Local, existe uma $\mathcal{L}$-coloração de $G$.

\subsubsection{Uma conjectura de K. Ohba}

Já dissemos que, em geral, o número cromático não está relacionado com o número lista-cromático. Porém, em situações bem particulares, pode ser que o número cromático esteja extremamente amarrado com o número listacromático. Existe uma conjectura muito interessante que ilustra isso:

Conjectura 4 (Ohba [13]): Se um grafo $G$ tem no máximo $2 \chi(G)+1$ vértices, então $\operatorname{ch}(G)=\chi(G)$.

Os próximos resultados são devidos a B. Reed e B. Sudakov. Em [15], os autores apresentam uma prova de que a Conjectura 4 é assintoticamente correta. O que vem a seguir não será o resultado propriamente contido em [15], mas uma versão anterior, que mostra algo um pouco mais fraco que a Conjectura 4.

Lema 13 (Reed e Sudakov [16]): Se o número lista-cromático de um grafo $G$ é estritamente maior que um inteiro $t$, então existe um conjunto de listas $\mathcal{L}=\{\mathcal{L}(v): v \in V(G)\}$ tal que cada lista desse conjunto tem tamanho pelo menos t, $G$ não é $\mathcal{L}$-colorível e o total de cores utilizadas nas listas é menor que $n:=|V(G)|$.

Prova. Suponha que $\operatorname{ch}(G)>t$ e escolha listas de cores $\mathcal{L}(v), v \in V(G)$, cada uma delas com tamanho pelo menos $t$, de forma que $G$ não seja $\mathcal{L}$-colorível, e de forma a minimizar $|\mathcal{C}|$, em que

$$
\mathcal{C}=\bigcup_{v \in V(G)} \mathcal{L}(v)
$$

Se $|\mathcal{C}|<n$, não há nada a fazer. Suponha, então, por absurdo, que $|\mathcal{C}| \geq n$, e construa um grafo $(\mathcal{C}, V(G))$-bipartido $H$ ligando uma cor $c \in \mathcal{C}$ a um vértice $v \in V(G)$ se $c \in \mathcal{L}(v)$. Tome um conjunto minimal $\mathcal{B} \subseteq \mathcal{C}$ tal que não existe um emparelhamento em $H$ que cobre $\mathcal{B}$. Note que um conjunto assim existe, pois $|\mathcal{C}| \geq n$. Pelo Teorema de Hall e pela escolha de $\mathcal{B}$, sabemos que 
$\left|\Gamma_{H}(\mathcal{B})\right|=|\mathcal{B}|-1$, e que existe um emparelhamento $M$ em $H\left[\mathcal{B} \cup \Gamma_{H}(\mathcal{B})\right]$ cobrindo $\Gamma_{H}(\mathcal{B})$. Disso decorre também que $|\mathcal{B}| \leq n$. Caso contrário, $M$ seria um emparelhamento cobrindo $V(G)$, e portanto teríamos uma $\mathcal{L}$-coloração de $G$ onde todos os vértices recebem cores diferentes.

Seja $w \in V(G) \backslash \Gamma_{H}(\mathcal{B})$. Troque as listas dos vértices em $\Gamma_{H}(\mathcal{B})$ pela lista de $w$. Esse novo conjunto de listas usa, no total, menos que $|\mathcal{C}|$ cores, e portanto existe uma coloração $\alpha$ de $G$ que respeita as novas listas. Mas então, trocando as cores atribuídas por $\alpha$ dos vértices em $\Gamma_{H}(\mathcal{B})$ pelas cores associadas a eles por $M$, temos uma coloração de $G$ que respeita as listas originais: absurdo.

Lema 14 (Reed e Sudakov [16]): Seja $k>1$ um inteiro e $G$ um grafo $3 k$ partido, com no máximo $5 k$ vértices e pelo menos $2 k$ partes de tamanho 1 . Então $\operatorname{ch}(G) \leq 3 k$.

Prova. A prova vai ser probabilística. Chame $s_{i}, 1 \leq i \leq 2 k$, aos vértices de $2 k$ partes unitárias escolhidas arbitrariamente. Denote as partes restantes por $U_{j}, 1 \leq j \leq k$. Seja $\mathcal{L}$ um conjunto de listas de cores, uma para cada vértice, e cada uma com tamanho pelo menos $3 k$. No caso de uma lista possuir mais que $3 k$ cores, truncamos essa lista para que ela tenha exatamente $3 k$ cores. Suponha que $G$ não seja $\mathcal{L}$-colorível. Pelo Lema 13 , podemos supor que o total de cores nessas listas é menor que $n:=|V(G)| \leq 5 k$. Portanto, para cada par de vértices $u \neq v$, temos que $|\mathcal{L}(u) \cap \mathcal{L}(v)|=|\mathcal{L}(u)|+|\mathcal{L}(u)|-$ $|\mathcal{L}(u) \cup \mathcal{L}(v)| \geq 3 k+3 k-5 k=k$.

A seguir, vamos construir $k$ conjuntos disjuntos $C_{1}, \ldots, C_{k}$, cada um deles contendo exatamente 3 cores, em que cada $C_{i}$ satisfaz a seguinte propriedade: para cada par de cores em $C_{i}$, existe um modo de colorir $s_{2 i-1}$ e $s_{2 i}$ com essas duas cores respeitando as listas de ambos os vértices. A construção desses conjuntos é feita do seguinte modo. Cada conjunto $C_{i}$ conterá as cores $p_{i}, q_{i}$ e $r_{i}$, onde $p_{i} \in \mathcal{L}\left(s_{2 i-1}\right) \cap \mathcal{L}\left(s_{2 i}\right)$ é escolhida de modo a ser diferente de toda cor $p_{j}, j<i$. Note que isso é possível pois $\left|\mathcal{L}\left(s_{2 i-1}\right) \cap \mathcal{L}\left(s_{2 i}\right)\right| \geq k$. A cor $q_{i} \in \mathcal{L}\left(s_{2 i-1}\right)$ é escolhida de modo a ser diferente de $q_{j}, j<i$, e de todas as cores $p_{j}, j \leq k$. A cor $r_{i} \in \mathcal{L}\left(s_{2 i}\right)$ é escolhida de modo a ser diferente de $r_{j}$, $j<i$, de todas as cores $q_{j}, j \leq k$, e de todas as cores $p_{j}, j \leq k$. Durante as escolhas mencionadas anteriormente, não haverá falta de cores pois a lista de cada vértice tem $3 k$ elementos.

Vamos colorir $G$ em duas rodadas. Na primeira, sorteamos uma cor $t_{i} \in$ $C_{i}$ uniformemente e independente para cada $1 \leq i \leq k$. Com as cores 
em $C_{i}-t_{i}$, colorimos os vértices $s_{2 i-1}$ e $s_{2 i}$ respeitando suas listas. Também sorteamos uma permutação $\pi:[k] \longrightarrow[k]$ com probabilidade uniforme dentre todas as permutações de $k$ elementos. A seguir, colorimos com cor $t_{\pi(i)}$ todos os vértices de $U_{i}$ que possuem $t_{\pi(i)}$ em sua lista. Ao final desta primeira rodada, pode ser que ainda sobraram alguns vértices não coloridos. Ponha $\mathcal{C}=\bigcup_{i} C_{i}$, e denote por $V^{\prime}$ o conjunto de todos os vértices não coloridos ao final da primeira rodada. Ponha $\mathcal{L}^{\prime}(v)=\mathcal{L}(v) \backslash \mathcal{C}$ para todo $v \in V^{\prime}$. Note que se $\mathcal{L}(v)=\mathcal{C}$ para algum vértice $v$, então $v$ já foi colorido na primeira rodada, e portanto nenhum vértice em $V^{\prime}$ possui lista vazia.

Observe que, dados $1 \leq i \leq k$ e $c \in \mathcal{C}$, a probabilidade de que $t_{\pi(i)}=c$ é exatamente $(1 / 3)(1 / k)=1 / 3 k=1 /|\mathcal{C}|$. Para cada vértice $v \in \bigcup_{i} U_{i}$ associe uma variável aleatória $X_{v}$ que vale $1 /\left|\mathcal{L}^{\prime}(v)\right|$ se $v \in V^{\prime}$ e 0 caso contrário. Podemos então calcular a probabilidade de que um vértice $v \in U_{i}$ pertença a $V^{\prime}$ :

$$
\begin{aligned}
\operatorname{Pr}\left(v \in V^{\prime}\right) & =\operatorname{Pr}\left(t_{\pi(i)} \notin \mathcal{L}(v) \cap \mathcal{C}\right)=1-\operatorname{Pr}\left(t_{\pi(i)} \in \mathcal{L}(v) \cap \mathcal{C}\right) \\
& =1-\frac{|\mathcal{L}(v) \cap \mathcal{C}|}{|\mathcal{C}|}=1-\frac{|\mathcal{L}(v)|-\left|\mathcal{L}^{\prime}(v)\right|}{|\mathcal{C}|} \\
& =1-\frac{|\mathcal{C}|-\left|\mathcal{L}^{\prime}(v)\right|}{|\mathcal{C}|}=\frac{\left|\mathcal{L}^{\prime}(v)\right|}{|\mathcal{C}|}
\end{aligned}
$$

Assim, podemos delimitar o valor esperado da soma de todas variáveis $X_{v}$. Ponha $U=\bigcup_{i} U_{i}$. Temos

$$
\begin{aligned}
\mathbf{E}\left(\sum_{v \in U} X_{v}\right) & =\sum_{v \in U} \mathbf{E}\left(X_{v}\right)=\sum_{v \in U} \frac{\operatorname{Pr}\left(v \in V^{\prime}\right)}{\left|\mathcal{L}^{\prime}(v)\right|}=\sum_{v \in U} \frac{\left|\mathcal{L}^{\prime}(v)\right|}{\left|\mathcal{L}^{\prime}(v)\right| \cdot|\mathcal{C}|} \\
& =\sum_{v \in U} \frac{1}{|\mathcal{C}|}=\frac{|U|}{|\mathcal{C}|} \leq \frac{5 k-2 k}{3 k}=1 .
\end{aligned}
$$

Pelo princípio do primeiro momento, existe uma escolha das cores $t_{i}$ e da permutação $\pi$ de forma que a soma $\sum_{v \in U} X_{v}$ seja no máximo 1. Fixe uma tal escolha dos $t_{i}$ 's e da permutação $\pi$. Sejam $v_{1}, \ldots, v_{\ell}$ os vértices de $V^{\prime}$, e suponha sem perda de generalidade que, se $i<j$, então $\left|\mathcal{L}^{\prime}\left(v_{i}\right)\right| \leq\left|\mathcal{L}^{\prime}\left(v_{j}\right)\right|$. Afirmamos que para todo vértice $v_{i} \in V^{\prime}$, temos $\left|\mathcal{L}^{\prime}\left(v_{i}\right)\right| \geq i$. De fato, suponha que isso não aconteça. Seja $v_{j}$ o primeiro vértice para o qual $\left|\mathcal{L}^{\prime}\left(v_{j}\right)\right|<j$. Mas então, temos um absurdo:

$$
\sum_{v \in U} X_{v}=\sum_{v \in V^{\prime}} X_{v}=\sum_{i=1}^{\ell} X_{v_{i}} \geq \sum_{i=1}^{j} X_{v_{i}}=\sum_{i=1}^{j} \frac{1}{\left|\mathcal{L}^{\prime}\left(v_{i}\right)\right|} \geq \frac{j}{j-1}>1 .
$$


Agora, para cada $v_{i} \in V^{\prime}$, escolha uma cor de sua lista que seja diferente das cores já escolhidas para $v_{j}, 1 \leq j<i$. Note que sempre podemos fazer tal escolha pelos motivos apresentados no parágrafo anterior. Portanto, ao contrario da hipótese que adotamos no início da prova, $G$ é $\mathcal{L}$-colorível. Assim completamos a prova do lema.

Teorema 15 (Reed e Sudakov [16]): Seja $G$ um grafo. Se $|V(G)| \leq \frac{5}{3} \chi(G)-$ $\frac{4}{3}$, então $\operatorname{ch}(G)=\chi(G)$.

Prova. Suponha, por contradição, que a afirmação seja falsa. Seja $G$ um contra-exemplo com o número mínimo de vértices e, dentre esses, com o número máximo de arestas. Note que esse grafo deve ser um grafo $\chi(G)$ partido completo pois adicionando-se uma aresta entre classes de uma coloração ótima de $G$ produz-se um novo contra-exemplo. Pelo Lema 13, existe um conjunto de listas $\mathcal{L}=\{\mathcal{L}(v): v \in V(G)\}$ usando no total menos que $|V(G)|$ cores, cada lista com tamanho pelo menos $\chi(G)$, tal que $G$ não é $\mathcal{L}$-colorível.

Afirmamos que nenhum conjunto estável maximal em $G$ possui tamanho exatamente 2. Do contrário, seja $S=\{x, y\}$ um conjunto estável maximal de tamanho 2 em $G$. Note que $G-S$ possui $|V(G)|-2$ vértices. Como $G$ é $3 k$-partido completo e $S$ é um conjunto estável maximal, $G-S$ tem número cromático $\chi(G)-1$. Esses dois fatos implicam que $G-S$ satisfaz a hipótese do teorema:

$$
\begin{aligned}
|V(G-S)| & =|V(G)|-2 \leq\left(\frac{5}{3} \chi(G)-\frac{4}{3}\right)-2=\frac{5}{3}(\chi(G)-1)-\frac{5}{3} \\
& <\frac{5}{3} \chi(G-S)-\frac{4}{3} .
\end{aligned}
$$

Pela escolha de $G$, sabemos que $\operatorname{ch}(G-S)=\chi(G-S)=\chi(G)-1$. Lembrando que cada lista tem tamanho no mínimo $\chi(G) \geq|V(G)| / 2$, e que o número de cores na união das listas é menor que $|V(G)|$, temos que $\mathcal{L}(x) \cap \mathcal{L}(y) \neq \emptyset$. Seja $c$ uma cor em $\mathcal{L}(x) \cap \mathcal{L}(y)$. Ponha $\mathcal{L}^{\prime}=\{\mathcal{L}(v) \backslash\{c\}: v \in V(G)\}$. Tome uma $\mathcal{L}^{\prime}$-coloração própria de $G-S$ e complete-a a uma $\mathcal{L}$-coloração própria de $G$ colocando cor $c$ em $x$ e $y$. Isto é um absurdo pois $G$ não é $\mathcal{L}$-colorível. Então $G$ não possui conjuntos estáveis maximais de tamanho 2 .

Sejam $r=\chi(G) \bmod 3$, e $k=(\chi(G)-r) / 3$. Cada lista em $\mathcal{L}$ tem tamanho pelo menos $3 k+r$. Temos no máximo $\frac{5}{3} \chi(G)-\frac{4}{3}=5 k+\frac{5}{3} r-\frac{4}{3} \leq$ $5 k+r$ vértices. Chamemos de $x$ o número de partes unitárias de $G$. Como cada parte não unitária tem pelo menos 3 vértices, temos que $x+3(3 k+r-$ 
$x) \leq 5 k+r$. Isso implica que o número de partes unitárias é no mínimo $2 k+r$. Pinte $r$ partes unitárias quaisquer com $r$ cores distintas, cada qual retirada da lista do respectivo vértice; remova essas cores das listas dos outros vértices. Ponha $W$ como sendo a união dessas $r$ partes. Retire $W$ de $G$. O que sobra é um grafo $3 k$-partido, com no máximo $5 k$ vértices, com no mínimo $2 k$ partes unitárias e uma lista $\mathcal{L}^{\prime}(v)$ com no mínimo $3 k$ cores para cada vértice $v$. Pelo Lema $14, G-W$ possui uma $\mathcal{L}^{\prime}$-coloração. Podemos estender essa coloração a uma $\mathcal{L}$-coloração de $G$ da maneira óbvia. Isto é um absurdo, e portanto o teorema segue.

\subsection{Coloração total de grafos}

A seguir vamos apresentar dois limitantes para o número cromático total. Eles são indicadores de que a conjectura da coloração total deve ser verdadeira. Todos os resultados foram retirados de [12].

Teorema 16: Para todo grafo $G$ com $n$ vértices e grau máximo $\Delta$, temos $\chi_{T}(G) \leq \Delta+\lceil\log n\rceil+3$.

Prova. Fixe uma $(\Delta+1)$-coloração $\alpha$ das arestas, isto é, um conjunto de emparelhamentos disjuntos $\left\{M_{1}, M_{2}, \ldots, M_{\Delta+1}\right\}$ cobrindo as arestas do grafo. O Teorema de Vizing garante a existência de tal coloração. Escolha uma coloração $\varphi$ dos vértices de $G$ com cores $1,2, \ldots, \Delta+1$ como garante o método da coloração gulosa. Considere todas as $(\Delta+1)$-colorações $\varphi_{1}, \varphi_{2}, \ldots, \varphi(\Delta+1)$ ! que são obtidas de $\varphi$ permutando-se as classes de coloração (os nomes das cores). Note que podemos escolher a coloração $\varphi$ de modo a usar todas as $(\Delta+1)$ cores disponíveis. Isso evita que existam duas colorações $\varphi_{i}$ e $\varphi_{j}$, $i \neq j$, que sejam idênticas.

Ao escolhermos uma coloração $\varphi_{i}$ para os vértices, pode acontecer que alguma aresta tenha a mesma cor que uma de suas pontas. Uma aresta assim é chamada de inconveniente. Seja $R_{i}$ o grafo de rejeição da coloração $\varphi_{i}$, isto é, o subgrafo de $G$ gerado pelas arestas inconvenientes. Vamos provar que é possível escolher uma coloração $\varphi_{i}$ de modo que o número de arestas inconvenientes incidentes em cada vértice seja no máximo $\ell:=\lceil\log n\rceil+1$, isto é, de modo que $R_{i}$ tenha grau máximo $\ell$. Se conseguirmos fazer isso, basta usarmos $\ell+1$ novas cores para recolorir as arestas de $R_{i}$ (usando o Teorema de Vizing novamente) e o grafo já estará totalmente $(\Delta+\lceil\log n\rceil+3)$-colorido. 
Sorteie uma coloração $\psi=\varphi_{i}$ uniformemente dentre as $(\Delta+1)$ ! colorações acima consideradas. Sorteada $\psi$, uma aresta $v w$ em $\nabla(v)$ pode ser inconveniente por dois motivos: porque sua cor é $\psi(v)$, ou porque sua cor é $\psi(w)$. Se a cor de $v w$ é $\psi(w)$, dizemos que $v w$ é $v$-inconveniente. Note que no máximo uma aresta em $\nabla(v)$ possui cor $\psi(v)$. Todas as demais arestas inconvenientes em $\nabla(v)$ possuem cores diferente de $\psi(v)$, e são, portanto, arestas $v$-inconvenientes. Considere o seguinte evento $A_{v}$ : existem $\ell$ arestas $v$-inconvenientes em $\nabla(v)$. Nossa estratégia será limitar a probabilidade desse evento e aplicar a Desigualdade de Markov.

Fixe um conjunto $U:=\left\{v u_{1}, v u_{2}, \ldots, v u_{\ell}\right\}$. Claramente todas as arestas em $U$ possuem cores duas a duas distintas. Portanto, se $\varphi\left(u_{i}\right)=\varphi\left(u_{j}\right)$, para algum par de índices $1 \leq i<j \leq \ell$, a probabilidade de que todas as arestas em $U$ sejam $v$-inconvenientes é zero. Senão, temos que $(\Delta-\ell+1) ! /(\Delta+1)$ ! é um limitante para essa probabilidade. Pela cota da união, a probabilidade de ocorrer $A_{v}$ é no máximo

$$
\left(\begin{array}{c}
\Delta \\
\ell
\end{array}\right) \frac{(\Delta-\ell+1) !}{(\Delta+1) !}<\frac{1}{\ell !}
$$

Mas $\ell !=(\lceil\log n\rceil+1) !>2^{\log n}=n$ se $n \geq 3$. Então, a probabilidade de que algum $\ell$-subconjunto de $\nabla(v)$ seja um conjunto de arestas $v$-inconvenientes é menor que $1 / n$. Usando a cota da união,

$$
\operatorname{Pr}\left(\bigcup_{v \in V(G)} A_{v}\right)<1 .
$$

Assim, com probabilidade positiva, nenhum evento $A_{v}$ ocorre. Ou seja, existe uma coloração $\varphi_{j}$ tal que $\Delta\left(R_{j}\right) \leq \ell$. Observe que a desigualdade não é estrita, pois cada vértice $v$ é possivelmente incidente em uma aresta adicional, que é inconveniente mas não $v$-inconveniente. Usando apenas $\ell+1$ novas cores, podemos recolorir o grafo $R_{j}$, e terminar de colorir totalmente o grafo $G$. Portanto, $\chi_{T}(G) \leq \Delta+\lceil\log n\rceil+3$.

Teorema 17: Para todo grafo $G$ com grau máximo $\Delta$ suficientemente grande, temos $\chi_{T}(G) \leq \Delta+2 \Delta^{\frac{3}{4}}$.

Prova. Fixe uma coloração $\alpha$ das arestas usando cores $0,1, \ldots, \Delta$, isto é, um conjunto de emparelhamentos disjuntos $M_{0}, \ldots, M_{\Delta}$ cobrindo todas as arestas. Sejam

$$
k=\left\lceil\Delta^{\frac{1}{3}}\right\rceil \quad \text { e } \quad \ell=\left\lfloor\frac{\Delta+\Delta^{\frac{3}{4}}}{k}\right\rfloor .
$$


Vamos particionar os vértices de $G$ em $k$ classes $V_{1}, \ldots, V_{k}$ tais que cada vértice $v \in V(G)$ tem menos que $\ell$ vizinhos em cada classe. Queremos colorir cada classe $V_{i}$ usando as cores em $C_{i}=\{(i-1) \ell, \ldots, i \ell-1\}$. Isso produziria uma $k \ell$ coloração total de $G$. No entanto, as cores dadas aos vértices podem conflitar com as cores das arestas. Dizemos que uma aresta e é inconveniente se $e \in M_{i}$ e uma das pontas de $e$ pertence a $V_{i}$. Note que uma aresta pode ser inconveniente mesmo que possua uma cor diferente das cores atribuídas às suas pontas. Além disso, dizemos que uma aresta $e=u v$ é $v$-inconveniente se $e \in M_{i}$ e $u \in V_{i}$. Vamos definir "inconveniência" desse modo amplo para facilitar a análise.

Queremos provar que é possível particionar $V(G)$ em $k$ classes $V_{1}, \ldots, V_{k}$ de forma que:

(i) para cada vértice $v$ e classe $i,\left|V_{i} \cap \Gamma(v)\right|<\ell$;

(ii) para cada vértice $v$, existem no máximo $\left\lfloor\Delta^{\frac{3}{4}}-2\right\rfloor$ arestas $v$-inconvenientes.

Vamos argumentar que isso é suficiente para podermos colorir totalmente o grafo com o número de cores desejado.

Inicialmente, podemos colorir os vértices de cada classe $V_{i}$ usando as cores em $C_{i}$. Isso é possível graças a (i), que força o grafo induzido por cada $V_{i}$ a possuir grau máximo $\ell-1$. As $k \ell$ cores usadas nesse processo são $0,1, \ldots, k \ell-1$, sendo $k \ell \leq \Delta+\Delta^{\frac{3}{4}}$.

Dentre as arestas inconvenientes, vamos apenas considerar aquelas que realmente possuem a mesma cor que uma de suas pontas. Temos no máximo $\left\lfloor\Delta^{\frac{3}{4}}-1\right\rfloor$ dessas arestas incidindo em cada vértice $v\left(\left\lfloor\Delta^{\frac{3}{4}}-2\right\rfloor\right.$ arestas $v$ inconvenientes e possivelmente uma aresta extra que recebeu a mesma cor que $v$ ). Podemos então colorí-las usando no máximo $\left\lfloor\Delta^{\frac{3}{4}}\right\rfloor$ novas cores, pois o grafo gerado por essas arestas tem grau no máximo $\left\lfloor\Delta^{\frac{3}{4}}-1\right\rfloor$. Assim, conseguimos uma coloração total de $G$ que usa no máximo $\left\lfloor\Delta+2 \Delta^{\frac{3}{4}}\right\rfloor$ cores.

Para provar a existência de uma partição satisfazendo (i) e (ii), vamos sortear a classe de cada vértice uniforme e independentemente. Seja $A_{v, i}$ o evento em que o vértice $v$ possui pelo menos $\ell$ vizinhos na classe $i$ (viola a condição (i)). Seja $B_{v}$ o evento em que o vértice $v$ viola a condição (ii). Se conseguirmos evitar todos os eventos $A_{v, i}$ e todos os eventos $B_{v}$, então estamos feitos.

Vamos primeiro delimitar a probabilidade desses eventos. Considere a variável aleatória $X_{v, i}$ que conta o número de vizinhos de $v$ que caem na classe $i$. Observe que $A_{v, i}$ ocorre se e somente se $X_{v, i} \geq \ell$. Como cada variável 
da forma $X_{v, i}$ pode ser escrita como soma de no máximo $\Delta$ variáveis $\{0,1\}$, podemos usar o Limitante de Chernoff para achar um limitante superior para $\operatorname{Pr}\left(X_{v, i} \geq \ell\right)$. Temos, então, que

$$
\begin{aligned}
\operatorname{Pr}\left(A_{v, i}\right) & \leq \operatorname{Pr}\left(X_{v, i} \geq \ell\right) \\
& =\operatorname{Pr}\left(\operatorname{BIN}\left(\Delta, \frac{1}{k}\right) \geq l\right) \\
& \leq \operatorname{Pr}\left(\operatorname{BIN}\left(\Delta, \frac{1}{k}\right) \geq \frac{\Delta+\Delta^{\frac{3}{4}}}{k}\right) \\
& \leq \operatorname{Pr}\left(\left|\operatorname{BIN}\left(\Delta, \frac{1}{k}\right)-\frac{\Delta}{k}\right|>\Delta^{\frac{5}{12}}\right) \\
& \leq 2 \exp \left\{-\Delta^{\frac{10}{12}} / 3 \Delta^{\frac{2}{3}}\right\} \\
& \leq 2 e^{-\frac{1}{3} \Delta^{\frac{1}{6}}} .
\end{aligned}
$$

Da mesma forma, podemos superestimar o número de arestas $v$-inconvenientes por uma binomial de parâmetros $\Delta$ e $\frac{1}{k}$. Temos, então, que

$$
\begin{aligned}
\operatorname{Pr}\left(B_{v}\right) & \leq \operatorname{Pr}\left(\operatorname{BIN}\left(\Delta, \frac{1}{k}\right)>\Delta^{\frac{3}{4}}-1\right) \\
& <\operatorname{Pr}\left(\left|\operatorname{BIN}\left(\Delta, \frac{1}{k}\right)-\frac{\Delta}{k}\right|>\frac{\Delta}{k}\right) \\
& <2 e^{-\frac{1}{3} \Delta^{\frac{2}{3}}} .
\end{aligned}
$$

As probabilidades de ambos os eventos decrescem exponencialmente com $\Delta$ e são no máximo $p=2 e^{-\frac{1}{3} \Delta^{1 / 6}}$. Agora vamos limitar a dependência desses eventos para poder aplicar o Lema Local. Repare que $A_{v, i}$ e $B_{v}$ são mutuamente independentes de todos os eventos $A_{u, j}$ e $B_{u}$ se o vértice $u$ se encontra a uma distância pelo menos 3 de $v$. Assim, cada evento ruim é independente de todos os outros exceto no máximo $d=(k+1) \times\left(\Delta^{2}+\Delta+1\right) \leq \Delta^{3}$ eventos. Mas $4 p d<1$ para $\Delta$ suficientemente grande. Então, pelo Lema Local, sabemos que existe um modo de escolher a partição $V_{1}, \ldots, V_{k}$ de modo a satisfazer as condições (i) e (ii). E, portanto, é possível colorir $G$ totalmente com $\Delta+2 \Delta^{\frac{3}{4}}$ cores, desde que $\Delta$ seja suficientemente grande. 


\section{Capítulo 6}

\section{Grafos Livres de Triângulos}

\subsection{Introdução}

No Capítulo 3, mostramos o Teorema de Brooks, que é um limitante para o número cromático de grafos que satisfazem algumas poucas condições. Ele é justo, no sentido de que podemos exibir infinitos grafos que satisfazem as hipóteses do teorema e que possuem $\chi(G)=\Delta(G)$. Uma pergunta natural que surge é saber se podemos melhorar esse limitante, quando o conjunto de grafos é mais restrito.

De acordo com [12], Vizing em [18] foi o primeiro a perguntar-se sobre a existência de limitantes melhores para o número cromático quando os grafos são livres de triângulos, isto é, não contêm $K_{3}$ como subgrafo. A pergunta pode parecer gratuita, mas não é: a existência de cliques de tamanho $k$ exige que número cromático seja pelo menos $k$; portanto a ausência de cliques de tamanho $3(4,5,6, \ldots)$ deve, aparentemente, fazer com que o limitante obtido pelo Teorema de Brooks pareça muito relaxado. O primeiro resultado nessa linha foi obtido independentemente por Borodin e Kostochka [5], Catlin [6] e Lawrence [9]. Eles mostraram que grafos livres de $K_{4}$ possuem número cromático no máximo $\frac{3}{4}(\Delta+2)$. Muitos anos depois, Johansson [7] mostrou o seguinte:

Teorema 18: Existe um $\Delta_{0}$ tal que todo grafo $G$, livre de triângulos, com grau máximo $\Delta \geq \Delta_{0}$, tem $\chi(G) \leq \frac{160 \Delta}{\ln \Delta}$. 


\subsection{Uma intuição}

Antes de provar qualquer resultado, vamos tentar dar uma idéia da razão pela qual é possível colorir um grafo livre de triângulos com apenas $\frac{\Delta}{\ln \Delta}$ cores. Suponha que estamos usando um conjunto $C$ de cores para colorir os vértices de um grafo $G$ livre de triângulos. Olhe para a vizinhança de um vértice $v$ arbitrário. Note que $\Gamma(v)$ é um conjunto estável.

Vamos sortear, independentemente para cada $u \in \Gamma(v)$, uma cor escolhida com probabilidade uniforme em $C$. Podemos tentar descobrir para que tamanhos de $C$ existe, com alta probabilidade, uma cor de $C$ que não foi atribuída a nenhum vizinho de $v$. É claro que essa é uma situação muito particular: sorteamos apenas as cores de um pequeno grupinho de vértices do grafo $G$. Se quiséssemos fazer isso para toda vizinhança em $G$, nós iríamos, provavelmente, ficar bem longe de uma coloração própria de $G$.

No entanto, a idéia de sortear cores e olhar para a vizinhança de vértices aparece repetidas vezes na demonstração de um teorema de J. H. Kim [8] que vamos apresentar na Seção 6.4 abaixo. O teorema não é propriamente sobre grafos livres de triângulos. Na verdade, ele prova uma cota para o número cromático com o mesmo sabor daquela do Teorema de Johansson, mas somente para grafos com cintura pelo menos 5 .

Dado que existe uma grande conexão entre o problema que mencionamos e o Teorema de Kim, vale a pena estudarmos o quão grande deve ser $C$ a fim de que sobre, com alta probabilidade, uma cor para usarmos em $v$.

\section{O problema do colecionador de cupons}

O problema que foi mencionado acima é bem estudado, e vamos dar-lhe uma aparência que tradicionalmente possui na literatura.

Suponha que existem $N$ tipos de figurinha, e que queremos comprar figurinhas até obter uma de cada tipo, isto é, até preenchermos um álbum. As figurinhas vêm embaladas, e portanto não podemos escolhê-las antes de as comprar. Seja $X$ a variável aleatória que conta o número de figurinhas que somos obrigados a comprar até que o álbum esteja completo. Vamos calcular o valor esperado de $X$.

Essa esperança não é difícil de calcular, se quebrarmos a variável de interesse $X$ em uma soma de variáveis mais simples $X_{i}, i=0,1, \ldots, N-1$, onde cada $X_{i}$ conta o número de figurinhas que temos que comprar até obter uma figurinha de tipo diferente, dado que já temos $i$ tipos. Temos que 
$X_{i}$ é uma variável aleatória com distribuição geométrica de parâmetro $\frac{N-i}{N}$. Temos $X=X_{0}+\cdots+X_{N-1}$. Pela linearidade da esperança, temos que

$$
\mathbf{E}(X)=\mathbf{E}\left(\sum_{i=0}^{N-1} X_{i}\right)=\sum_{i=0}^{N-1} \mathbf{E}\left(X_{i}\right)=\sum_{i=0}^{N-1} \frac{N}{N-i}=\sum_{j=1}^{N} \frac{N}{j} \approx N \ln N .
$$

\section{Concentração por cima}

Vamos mostrar que a variável $X$ definida acima é concentrada, isto é, assume valores "longe" da média com probabilidade que tende a 0 com $N$. Primeiramente, suponha que compramos $(1+\epsilon) N \ln N$ figurinhas, e chame de $Y$ a variável aleatória que conta o número de tipos que ficam faltando para completarmos o álbum. Decomponha a variável $Y$ em uma soma de variáveis $Y_{1}, \ldots, Y_{N}$, onde

$$
Y_{i}= \begin{cases}1 & \text { se figurinha do tipo } i \text { está faltando } \\ 0 & \text { caso contrário. }\end{cases}
$$

Pela linearidade da esperança, temos

$$
\begin{aligned}
\mathbf{E}(Y) & =\mathbf{E}\left(\sum_{i=1}^{N} Y_{i}\right)=\sum_{i=1}^{N} \mathbf{E}\left(Y_{i}\right)=\sum_{i=1}^{N} \operatorname{Pr}\left(Y_{i}=1\right) \\
& =N\left(1-\frac{1}{N}\right)^{(1+\epsilon) N \ln N}<N \mathrm{e}^{-(1+\epsilon) \ln N}=\frac{1}{N^{\epsilon}} .
\end{aligned}
$$

Segue da desigualdade de Markov que $\operatorname{Pr}(Y>0) \leq \mathbf{E}(Y)<\frac{1}{N^{\epsilon}}$. Portanto, com probabilidade tendendo a 1 quando $N$ tende a infinito, o álbum está completo.

\section{Concentração por baixo}

Por outro lado, se compramos $(1-\epsilon) N \ln N$ figurinhas, então é muito provável que faltem figurinhas para completarmos o álbum. Se refizermos a conta da esperança de $Y$, veremos que, nesse caso, o sinal da desigualdade nos atrapalha. Porém, fazendo contas similares àquelas que serão feitas para estimar $\mathbf{E}\left(X_{v}^{\prime}\right)$ no Teorema 19 da próxima seção, obtemos

$$
\mathbf{E}(Y) \approx N^{\epsilon}
$$


Vamos provar que $Y$ é concentrada em torno da média. Vamos calcular a variância de $Y$ e aplicar a desigualdade de Chebyshev.

$$
\begin{aligned}
\sigma^{2} & =\mathbf{E}\left((Y-\mathbf{E}(Y))^{2}\right) \\
& =\sum_{i=1}^{N}\left(\mathbf{E}\left(Y_{i}^{2}\right)-\mathbf{E}^{2}\left(Y_{i}\right)\right)+\sum_{i \neq j}\left(\mathbf{E}\left(Y_{i} Y_{j}\right)-\mathbf{E}\left(Y_{i}\right) \mathbf{E}\left(Y_{j}\right)\right) \\
& \leq \sum_{i=1}^{N} \mathbf{E}\left(Y_{i}\right)+\sum_{i \neq j}\left[\left(1-\frac{2}{N}\right)^{(1-\epsilon) N \ln N}-\left(1-\frac{1}{N}\right)^{2(1-\epsilon) N \ln N}\right] \\
& =\mathbf{E}(Y)+\sum_{i \neq j}\left[\left(1-\frac{2}{N}\right)^{(1-\epsilon) N \ln N}-\left(1-\frac{2}{N}+\frac{1}{N^{2}}\right)^{(1-\epsilon) N \ln N}\right] \\
& \leq \mathbf{E}(Y) \\
& \leq N^{\epsilon}
\end{aligned}
$$

A Desigualdade de Chebyshev diz que $\operatorname{Pr}(|Y-\mathbf{E}(Y)| \geq \lambda \sigma) \leq \frac{1}{\lambda^{2}}$. Tomando $\lambda=N^{\epsilon / 2} / 2$, temos que $\operatorname{Pr}\left(|Y-\mathbf{E}(Y)| \geq N^{\epsilon} / 2\right) \leq 4 / N^{\epsilon}$. Assim, $Y=0$ com probabilidade que tende a 0 com $N$. Portanto, com alta probabilidade, restam muitos espaços no álbum a serem preenchidos.

\section{Aplicando a idéia}

No nosso caso, o álbum que (não) queremos preencher representa o conjunto de cores que temos disponíveis para colorir um vértice $v$ de um grafo. Cada tipo de figurinha representa uma cor desse conjunto. O número de figurinhas que vamos comprar está fixo e é igual ao número de vizinhos de $v$. A cada vizinho de $v$ que é colorido, temos de marcar essa cor para lembrarmos que ela não pode ser usada em $v$.

Suponha que $v$ é um vértice de grau máximo do grafo. Ponha $\left(1+\epsilon_{2}\right)=$ $1 /\left(1-\epsilon_{1}\right)$. Suponha que $|C| \approx\left(1+\epsilon_{2}\right) d(v) / \ln d(v)=\left(1+\epsilon_{2}\right) \Delta / \ln \Delta$. Trocando o denominador por $\ln |C|$, não estamos alterando significativamente o tamanho de $C$. Ou seja, $|C| \approx\left(1+\epsilon_{2}\right) \Delta / \ln |C|$. Portanto, $\Delta \approx(1-$ $\left.\epsilon_{1}\right)|C| \ln |C|$. Pela concentração por baixo, com alta probabilidade vão sobrar cores não marcadas em $C$ para usarmos em $v$. 


\subsection{Uma cota fraca}

Esta seção serve, ao mesmo tempo, para dar um exemplo de um limitante não trivial para o número cromático de grafos livres de triângulos, e para introduzir idéias que serão usadas na próxima seção. O resultado a seguir foi retirado de [12]. Aqui, apresentamos uma prova com detalhes de contas que são suprimidos na fonte bibliográfica.

Teorema 19: Se Gé um grafo livre de triângulos com grau máximo $\Delta$ suficientemente grande, então é possível colorir $G$ propriamente usando-se apenas $\left\lfloor\left(1-\frac{1}{2 \mathrm{e}^{6}}\right) \Delta\right\rfloor$ cores.

Prova. Primeiramente, suponha que $G$ é um grafo $\Delta$-regular. Podemos supor isso pois, caso $G$ não seja regular, existe uma construção muito simples que o transforma em um grafo regular sem aumentar o grau máximo e que mantém o grafo livre de triângulos. A construção é a seguinte: faça uma cópia do grafo e ligue cada vértice de grau menor que $\Delta$ a seu correspondente na cópia. O novo grafo obtido tem grau mínimo um maior que o grau mínimo do grafo original. Repita o procedimento até que o grau mínimo seja igual ao grau máximo. Note que $G$ é subgrafo do grafo resultante. Portanto, qualquer coloração própria do grafo resultante é também uma coloração própria de $G$.

A prova do teorema consiste em obter uma coloração parcial de $G$ que usa no máximo $\left\lfloor\left(1-\frac{1}{2 \mathrm{e}^{6}}\right) \Delta\right\rfloor$ cores de tal modo que na vizinhança de cada vértice não colorido existem pelo menos $\left\lceil\frac{\Delta}{2 \mathrm{e}^{6}}+1\right\rceil$ cores que aparecem mais de uma vez. Com isso somos capazes de usar o método da coloração gulosa para terminar de colorir o grafo, pois para cada vértice temos $\left\lfloor\left(1-\frac{1}{2 \mathrm{e}^{6}}\right) \Delta\right\rfloor$ cores para usar, e no máximo $\Delta-\left\lceil\left(\frac{\Delta}{2 \mathrm{e}^{6}}+1\right)\right\rceil=\left\lfloor\left(1-\frac{1}{2 \mathrm{e}^{6}}\right) \Delta\right\rfloor-1$ cores a evitar.

Começamos com um conjunto pequeno de cores $C=[\lfloor\Delta / 2\rfloor]$. Queremos ser capazes de garantir que a vizinhança de cada vértice tenha várias cores repetidas. Nosso procedimento de coloração consiste de duas etapas.

- Atribuição - Sorteamos independentemente, para cada vértice, uma cor de $C$ com probabilidade uniforme. Ao final desta etapa, teremos uma coloração $\varphi$ do grafo. Se $\varphi(v)=\varphi(u)$ e $u v \in E(G)$, dizemos que $v$ é inconveniente.

- Remoção - Descolorimos todos os vértices inconvenientes de $G$. Note que a finalidade principal desta etapa não é tornar própria a coloração 
$\varphi$, senão bastaria apagar a cor de alguns vértices inconvenientes. Aqui, estamos apagando as cores de todos os vértices inconvenientes para evitar que os eventos que serão considerados mais adiante tenham uma certa dependencia indesejada.

Quando, ao final deste processo, um vértice permaneceu colorido, dizemos que ele reteve a sua cor. Dizemos que uma cor é generosa com um vértice $v$ se ela foi retida por pelo menos dois vizinhos de $v$. Para cada vértice $v$, estamos interessados em que o número de cores generosas com $v$ seja grande. Mais especificamente, que esse número seja maior que $\left\lceil\frac{\Delta}{2 \mathrm{e}^{6}}\right\rceil$.

Para simplificar a análise, vamos considerar uma variável aleatória $X_{v}$ que conta o número de cores que foram atribuídas a pelo menos dois vizinhos de $v$ e retidas por todos eles. Para cada vértice $v$ seja $A_{v}$ o evento em que $X_{v}<\left\lceil\frac{\Delta}{2 \mathrm{e}^{6}}+1\right\rceil$. Seja $\mathcal{E}=\left\{A_{v}: v \in V(G)\right\}$ o conjunto dos eventos ruins que queremos evitar. Se conseguirmos evitar todos os eventos em $\mathcal{E}$, então teremos a coloração parcial desejada. Nossa estratégia será a seguinte:

(i) estudar a independência mútua entre os eventos em $\mathcal{E}$;

(ii) provar que $\mathbf{E}\left(X_{v}\right)$ é grande (suficientemente maior que $\frac{\Delta}{2 \mathrm{e}^{6}}$ ) para todo vértice $v$;

(iii) provar que os valores assumidos por $X_{v}$ estão concentrados em torno da média, isto é, que a probabilidade de $X_{v}<\left\lceil\Delta / 2 \mathrm{e}^{6}+1\right\rceil$ é pequena;

(iv) aplicar o Lema Local para concluir que é possível evitar todos os eventos em $\mathcal{E}$.

Para provar (i), observe que um evento $A_{v}$ depende somente das cores atribuídas a vértices que estão a uma distância no máximo 2 de $v$. Com isso, podemos concluir que $A_{v}$ é mutuamente independente de todos os eventos em $\mathcal{E}$ exceto dos eventos em $S_{v}:=\left\{A_{u}\right.$ : a distância entre $u$ e $v$ é menor que 5$\}$. Claramente $\left|S_{v}\right|<\Delta^{5}$ para todo $v \in V(G)$.

Agora, vamos provar (ii) que a esperança de $X_{v}$ é grande. Mais especificamente, vamos provar que:

$$
\mathbf{E}\left(X_{v}\right) \geq \frac{\Delta}{\mathrm{e}^{6}}-1
$$

Para calcularmos a esperança de $X_{v}$ considere a variável aleatória $X_{v}^{\prime}$ que conta o número de cores que foram atribuídas a exatamente 2 vizinhos de $v$ e retidas em ambos. Observe que $X_{v}^{\prime} \leq X_{v}$. Considere também as variáveis aleatórias $X_{c, u, w}=1$, para cada cor $c \in C$ e par $u, w \in \Gamma(v)$, onde $X_{c, u, w}=1$ 
se a cor $c$ é atribuída a $u$ e a $w$ e a mais nenhum outro vizinho de $v$, e $X_{c, u, w}=0$ caso contrário. Note que

$$
X_{v}^{\prime}=\sum_{c \in C} \sum_{\{u, w\} \subseteq \Gamma(v)} X_{c, u, w} .
$$

Devemos primeiro calcular a probabilidade de $X_{c, u, w}=1$. Para que $X_{c, u, w}$ seja 1, a cor $c$ deve ser atribuída a $u$ e a $w$ e não deve ser atribuída a ninguém $\operatorname{de} Z:=\Gamma(u) \cup \Gamma(w) \cup \Gamma(v) \backslash\{u, w\}$. Podemos afirmar que $|Z| \leq 3 \Delta-3 \leq 6|C|$. Portanto, a probabilidade de $X_{c, u, w}=1$ é pelo menos

$$
\frac{1}{|C|^{2}}\left(1-\frac{1}{|C|}\right)^{6|C|} \text {. }
$$

Lembrando que o grafo é livre de triângulos, temos $\left(\begin{array}{c}\Delta \\ 2\end{array}\right)$ possíveis escolhas do par $\{u, w\}$ e temos $|C|$ possíveis escolhas para a cor do par. Pela linearidade da esperança,

$$
\begin{aligned}
\mathbf{E}\left(X_{v}^{\prime}\right) & \geq|C|\left(\begin{array}{c}
\Delta \\
2
\end{array}\right)\left(\frac{1}{|C|^{2}}\right)\left(1-\frac{1}{|C|}\right)^{6|C|} \\
& \geq|C|^{2}(\Delta-1)\left(\frac{1}{|C|^{2}}\right)\left(1-\frac{1}{|C|}\right)^{6|C|} \\
& =(\Delta-1)\left(1-\frac{1}{|C|}\right)^{6|C|} .
\end{aligned}
$$

Agora, olhando para os três primeiros termos da expansão de Taylor de $\mathrm{e}^{-1 / y}$, é um fato conhecido que $e^{-1 / y} \leq 1-1 / y+1 / 2 y^{2}$. Usando isso, vamos achar um modo de subestimar $(1-1 / y)^{k}$ :

$$
\begin{aligned}
\left(1-\frac{1}{y}\right)^{k} & =\left(1-\frac{1}{y}+\frac{1}{2 y^{2}}-\frac{1}{2 y^{2}}\right)^{k} \geq\left(\mathrm{e}^{-\frac{1}{y}}-\frac{1}{2 y^{2}}\right)^{k} \\
& =\mathrm{e}^{-\frac{k}{y}}\left(1-\frac{\mathrm{e}^{-\frac{1}{y}}}{2 y^{2}}\right)^{k} \geq \mathrm{e}^{-\frac{k}{y}}\left(1-\frac{1}{y^{2}}\right)^{k}
\end{aligned}
$$

A última desigualdade vale, pois só estamos interessados em $y>1$. Aplicando a cota inferior acima, recursivamente, na cota encontrada para a esperança 
de $X_{v}^{\prime}$, podemos obter o seguinte:

$$
\begin{aligned}
\mathbf{E}\left(X_{v}^{\prime}\right) & \geq(\Delta-1)\left(1-\frac{1}{|C|}\right)^{6|C|} \geq(\Delta-1) \mathrm{e}^{-6}\left(1-\frac{1}{|C|^{2}}\right)^{6|C|} \\
& \geq(\Delta-1) \mathrm{e}^{-6} \mathrm{e}^{\frac{-6}{|C|}}\left(1-\frac{1}{|C|^{4}}\right)^{6|C|} \\
& \geq(\Delta-1) \mathrm{e}^{-6} \mathrm{e}^{\frac{-6}{|C|}} \mathrm{e}^{\frac{-6}{|C|^{3}}}\left(1-\frac{1}{|C|^{8}}\right)^{6|C|} \\
& \vdots \\
& \geq(\Delta-1) \mathrm{e}^{-6} \mathrm{e}^{\frac{-6}{|C|}} \mathrm{e}^{\frac{-6}{|C|^{3}}} \mathrm{e}^{\frac{-6}{|C|^{7}}} \mathrm{e}^{\frac{-6}{|C|^{\mid 5}}} \ldots \\
& =(\Delta-1) \mathrm{e}^{-6} \exp \left\{-6\left(\sum_{i=1}^{\infty} \frac{1}{|C|^{2^{i}-1}}\right)\right\} .
\end{aligned}
$$

Não é difícil de mostrar que $\sum_{i=1}^{\infty} 1 /|C|^{2^{i}-1} \leq 2 /|C|$ para $|C|$ suficientemente grande (isto é, para $\Delta$ suficientemente grande). Usando que $(1-x)<\mathrm{e}^{-x}$, temos que

$$
\begin{aligned}
\mathbf{E}\left(X_{v}^{\prime}\right) & \geq(\Delta-1) \mathrm{e}^{-6} \exp \left\{-\frac{12}{|C|}\right\} \geq(\Delta-1) \mathrm{e}^{-6}\left(1-\frac{12}{|C|}\right) \\
& \geq \frac{\Delta}{\mathrm{e}^{6}}-1 .
\end{aligned}
$$

Donde $\mathbf{E}\left(X_{v}\right) \geq \frac{\Delta}{\mathrm{e}^{6}}-1$, pois $X_{v}^{\prime} \leq X_{v}$.

Agora, temos que limitar a probabilidade de $X_{v}$ ser menor que $\left\lceil\Delta / 2 \mathrm{e}^{6}+1\right\rceil$ para provar que $X_{v}$ é concentrada em torno da média. Com isso, teremos conseguido (iii). Note que não podemos usar o limitante de Chernoff para mostrar que $X_{v}^{\prime}$ é concentrada pois, apesar de $X_{v}^{\prime}$ poder ser escrito como uma soma de variáveis 0-1, essas variáveis não seriam independentes. Escrevemos $X_{v}$ como soma de duas variáveis aleatórias $Y_{v}$ e $Z_{v}$. A variável $Y_{v}$ conta o número de cores atribuídas a pelo menos 2 vizinhos de $v$. A variável $Z_{v}$ conta o número de cores atribuídas a pelo menos 2 vizinhos de $v$ e apagadas de pelo menos um desses vértices. Note que

$$
X_{v}=Y_{v}-Z_{v}
$$

Nossa estratégia será provar que $Y_{v}$ e $Z_{v}$ são concentradas em torno da média e, usando isso, mostrar que $X_{v}$ é concentrada. 
Sabemos que $Y_{v}$ depende somente das cores atribuídas aos vizinhos de $v$. Alterando a cor de um único vizinho de $v$, mudamos $Y_{v}$ de no máximo $c=1$; se $Y_{v} \geq s$ então existem $2 s$ vizinhos de $v$ cujas cores certificam que $Y_{v} \geq s$. Com isso, podemos aplicar a Desigualdade de Talagrand e mostrar que, para $t \geq \sqrt{\Delta \log \Delta, \text { temos }}$

$$
\begin{aligned}
\operatorname{Pr}\left(\left|Y_{v}-\mathbf{E}\left(Y_{v}\right)\right|>t\right) & \leq 4 \exp \left\{-\frac{\left(t-60 \sqrt{2 \mathbf{E}\left(Y_{v}\right)}\right)^{2}}{16 \mathbf{E}\left(Y_{v}\right)}\right\} \\
& \leq 4 \mathrm{e}^{-(t / 2)^{2} / 16 \Delta} \\
& =4 \mathrm{e}^{-t^{2} / 64 \Delta}
\end{aligned}
$$

pois $\mathbf{E}\left(Y_{v}\right)<\Delta$, e estamos supondo $\Delta$ suficientemente grande.

A variável $Z_{v}$ se comporta da mesma forma. Sabemos que $Z_{v}$ depende das cores dadas a $v$, aos vizinhos de $v$ e aos vizinhos dos vizinhos de $v$. Alterando a cor de um único vértice desses, $Z_{v}$ é alterada de no máximo $c=1$; se $Y_{v} \geq s$ então existem $r s=3 s$ vértices cujas cores certificam que $Y_{v} \geq s$, a saber, para cada cor precisamos de dois vizinhos de $v$ e um terceiro vértice que recebeu a mesma cor e que é adjacente a eles. Com isso podemos aplicar a Desigualdade de Talagrand e mostrar que, para $t \geq \sqrt{\Delta \log \Delta}$ e $\Delta$ suficientemente grande, temos

$$
\begin{aligned}
\operatorname{Pr}\left(\left|Z_{v}-\mathbf{E}\left(Z_{v}\right)\right|>t\right) & \leq 4 \exp \left\{-\frac{\left(t-60 \sqrt{3 \mathbf{E}\left(Z_{v}\right)}\right)^{2}}{24 \mathbf{E}\left(Z_{v}\right)}\right\} \\
& \leq 4 \mathrm{e}^{-(t / 2)^{2} / 24 \Delta} \\
& =4 \mathrm{e}^{-t^{2} / 96 \Delta}
\end{aligned}
$$

pois $\mathbf{E}\left(Z_{v}\right)<\Delta$.

Agora que provamos que $Y_{v}$ e $Z_{v}$ estão concentradas em torno das respectivas médias, vamos provar que $X_{v}$ é concentrada. A propriedade da linearidade da esperança nos diz que $\mathbf{E}\left(X_{v}\right)=\mathbf{E}\left(Y_{v}\right)-\mathbf{E}\left(Z_{v}\right)$. Se $\left|X_{v}-\mathbf{E}\left(X_{v}\right)\right|>2 t$, então certamente $\left|Y_{v}-\mathbf{E}\left(Y_{v}\right)\right|>t$ ou $\left|Z_{v}-\mathbf{E}\left(Z_{v}\right)\right|>t$. Usando a cota da união, podemos dizer que a probabilidade de uma dessas duas coisas ocorrerem é limitada por

$$
4 \mathrm{e}^{-t^{2} / 96 \Delta}+4 \mathrm{e}^{-t^{2} / 64 \Delta}
$$

desde que $t \geq \sqrt{\Delta \log \Delta}$ e $\Delta$ seja suficientemente grande. Tomando $t=$ 


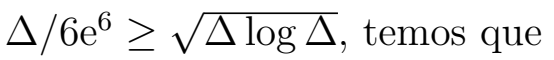

$$
\begin{aligned}
\operatorname{Pr}\left(\left|X_{v}-\mathbf{E}\left(X_{v}\right)\right|>2 t\right) & \leq 4 \mathrm{e}^{-t^{2} / 96 \Delta}+4 \mathrm{e}^{-t^{2} / 64 \Delta} \\
& =4 \mathrm{e}^{-\Delta / 5184 \mathrm{e}^{12}}+4 \mathrm{e}^{-\Delta / 3456 \mathrm{e}^{12}} \\
& <\frac{1}{4 \Delta^{5}} .
\end{aligned}
$$

Agora resta fazer (iv). Nesse ponto, sabemos que cada evento em $\mathcal{E}$ é mutuamente independente de todos os outros exceto um número $d<\Delta^{5}$ deles, e que a probabilidade de um evento $A_{v} \in \mathcal{E}$ ocorrer é menor que $p:=$ $\frac{1}{4 \Delta^{5}}$. Podemos, então, aplicar o Lema Local e concluir que, como $4 p d<1$, existe um modo de atribuir as cores aos vértices de $G$ sem que nenhum evento em $\mathcal{E}$ ocorra. Isso termina a prova do teorema.

\subsection{O Teorema de J. H. Kim}

O teorema se aplica a grafos com cintura pelo menos 5, um caso particular de grafos sem triângulos.

Teorema 20: Para todo $\epsilon>0$ existe $\Delta_{0}$ tal que, se $G$ tem cintura pelo menos 5 e grau máximo $\Delta \geq \Delta_{0}$, então

$$
\chi(G) \leq(1+\epsilon) \frac{\Delta}{\ln \Delta} .
$$

A demonstração deste teorema é longa e complexa. Por causa disso, nas primeiras sub-seções, vamos descrevê-la mais lentamente para que a idéia geral permaneça na mente do leitor enquanto ele estiver passando pelas diversas etapas da prova.

\subsubsection{Construindo o supergrafo regular}

Durante a prova, será conveniente trabalharmos com um grafo regular. Nesta sub-seção vamos descrever um método para construir um supergrafo $H$ de $G$ que seja $\Delta$-regular e possua cintura pelo menos 5 . Note que, ao encontrarmos um limitante superior para $\chi(H)$, estaremos também achando um limitante superior para $\chi(G)$.

Suponha que $F$ é um grafo com cintura pelo menos 5 e $\delta(F)<\Delta(F)$. Vamos descrever como construir um supergrafo $F^{\prime}$ de $F$, também com cintura 
pelo menos 5 , que possua $\Delta\left(F^{\prime}\right)=\Delta(F)$ e $\delta\left(F^{\prime}\right)=\delta(F)+1$. Note que isso é suficiente para construirmos o supergrafo $H$ de $G$ que queremos. Basta repetir essa construção várias vezes, partindo de $G$, até obter $H \operatorname{com} \delta(H)=$ $\Delta(H)=\Delta$, isto é, um grafo $\Delta$-regular. O número de vezes que temos que repetir essa construção é obviamente finito, pois a cada passo o grau mínimo se aproxima mais de $\Delta$.

A construção é a seguinte. Seja $S=\left\{v_{1}, \ldots, v_{s}\right\}$ o conjunto de vértices de grau menor que $\Delta$ em $G$. Considere $2 s$ cópias duas a duas disjuntas de $G$, digamos, $A_{1}, \ldots, A_{s}$ e $B_{1}, \ldots, B_{s}$. Para cada $1 \leq i \leq s$, seja $S_{i}=$ $\left\{a_{i, 1}, \ldots, a_{i, s}\right\}$ o conjunto de vértices correspondentes a $S$ em $A_{i}$. Para cada $1 \leq i \leq s$, seja $S_{i}^{\prime}=\left\{b_{i, 1}, \ldots, b_{i, s}\right\}$ o conjunto de vértices correspondentes a $S$ em $B_{i}$. Adicione uma aresta $\left\{a_{i, j}, b_{j, i}\right\}$ para todo $i, j \in[s]$. Desse modo, não criamos nenhum triângulo e nenhum circuito de tamanho 4 no novo grafo. Também não aumentamos o grau máximo do grafo. Essa é, portanto, a construção de que gostaríamos.

\subsubsection{O procedimento}

A demonstração deste teorema tem algo em comum com a do Teorema 19 da seção anterior: o procedimento aleatório de coloração. Vamos lembrar que esse procedimento consistia de duas etapas:

- Atribuição - nesta etapa, cada vértice recebe, independentemente, uma cor escolhida uniformemente de um conjunto pré-determinado de cores.

- Remoção - esta etapa consiste em apagar a cor de todo vértice que recebeu a mesma cor que algum vizinho seu.

O problema é que agora estamos usando um número muito reduzido de cores, a saber, $(1+\epsilon) \frac{\Delta}{\ln \Delta}$. Para enxergar o que acontece, fixe um vértice arbitrário. O número esperado de vizinhos que retêm suas cores após a segunda etapa é

$$
\Delta\left(1-\frac{\ln \Delta}{(1+\epsilon) \Delta}\right)^{\Delta} \approx \Delta^{\epsilon / 1+\epsilon}
$$

Podemos perceber que esse número é muito pequeno, e isso nos impede de colorir o restante dos vértices usando o método guloso. O que Kim faz é aplicar um procedimento de coloração semelhante várias vezes, cada uma delas em um subconjunto dos vértices ainda não coloridos. Quando uma 
iteração termina de ser executada, os vértices que foram coloridos durante a iteração que passou não terão suas cores alteradas nem apagadas.

Devemos tomar cuidado para que a cor de um vértice colorido em uma determinada iteração não entre em choque com as cores que podem ser atribuídas aos seu vizinhos em iterações futuras. Isso nos leva a proibir certas cores de aparecerem em alguns vértices. Esse controle é feito com o auxílio de listas. Cada vértice $v$ possui um conjunto $\mathcal{L}_{i}(v)$ das cores que são "válidas" para ele no início da $i$-ésima iteração. Essas listas não são definidas previamente porque o algoritmo de coloração é probabilístico. Só quando a iteração $i$ termina, somos capazes de dizer quem é $\mathcal{L}_{i+1}(v)$ para todo $v$.

Inicialmente, todas as cores podem ser usadas em qualquer vértice, e portanto, $\mathcal{L}_{1}(v)=\left\{1, \ldots,\left\lfloor(1+\epsilon) \frac{\Delta}{\ln \Delta}\right\rfloor\right\}$, para todo $v$. Quando atribuímos uma cor $c$ a um vértice $v$ numa iteração $i$, temos que remover a cor $c$ da lista de todos os vizinhos de $v$. Desse modo evitamos incompatibilidade de cores entre vértices coloridos em iterações diferentes. Note que $\mathcal{L}_{i+1}(v) \subseteq \mathcal{L}_{i}(v)$ para todo $i=1,2, \ldots$, pois a cada iteração só vamos remover cores da lista de cada vértice. Vamos descrever em detalhes as etapas do procedimento modificado que será utilizado. Abaixo, a probabilidade $\mathbb{P}_{i, v, c}$ será definida mais para frente, e $K$ é uma constante menor que 1 e que depende de $\epsilon$. A partir de agora, o conjunto de passos a seguir será chamado simplesmente de i-ésima iteração:

1. Ativação - Para cada vértice $v$ ainda não colorido do grafo, "ativamos" $v$ com probabilidade $K / \ln \Delta$.

2. Atribuição - Para cada vértice ativo $v$, escolhemos independente e uniformemente uma cor $c_{v} \in \mathcal{L}_{i}(v)$ e colorimos $v$ com cor $c_{v}$. Se $u$ e $v$ são vértices adjacentes que foram ativados e $c_{u}=c_{v}$, dizemos que $u$ (ou, por simetria, $v$ ) é um vértice inconveniente.

3. Para todo vértice $v$, fazemos $\mathcal{L}_{i+1}(v) \longleftarrow \mathcal{L}_{i}(v)$.

4. Limpeza - Para todo vértice ativo $v$, e para todo vizinho $u$ de $v$, fazemos $\mathcal{L}_{i+1}(u) \longleftarrow \mathcal{L}_{i+1}(u) \backslash\left\{c_{v}\right\}$.

5. Remoção - Para todo vértice ativo $v$, descolorimos $v$ caso $v$ seja inconveniente.

6. Normalização - Para cada vértice $v$ e para cada cor $c \in \mathcal{L}_{i+1}(v)$, com probabilidade $\mathbb{P}_{i, v, c}$, fazemos $\mathcal{L}_{i+1}(v) \longleftarrow \mathcal{L}_{i+1}(v) \backslash\{c\}$. 
É provável que nesse ponto fiquem muitas perguntas na mente do leitor. Por exemplo, na etapa 4 , removemos a cor $c_{v}$ da lista de um vizinho de $v$ sem nem mesmo ter certeza de que $v$ permanecerá colorido. É verdade que estamos perdendo algumas cores se fazemos isso, mas desse modo, as cores que são removidas da lista de um vértice durante o passo 5 de uma iteração qualquer dependem somente das cores atribuídas aos seus vizinhos no passo 2 dessa mesma iteração.

O passo 6 parece um puro desperdício de cores. O fato é que esse passo facilita muito as contas que teremos de fazer no futuro. Vamos escolher as probabilidades $\mathbb{P}_{i, v, c}$ de modo que, após esse passo, para todo vértice $v$ e cor $c \in \mathcal{L}_{i}(v)$, a probabilidade de que $c$ permaneça em $\mathcal{L}_{i+1}(v)$ seja a mesma. Veremos como fazer isso mais adiante, na Seção 6.4.5.

Assim como no Teorema 19, se depois do passo 6 um vértice ativo $v$ permaneceu colorido, dizemos que $v$ reteve a sua cor. Lembramos que, se um vértice reteve a sua cor em uma iteração passada, essa cor nunca mais será removida nem alterada.

\subsubsection{Objetivos}

Supor que podemos colorir o grafo todo somente executando repetidamente a iteração acima é querer demais. Temos que parar em algum ponto e fazer algo determinístico como foi feito no Teorema 19. Uma possibilidade seria executar o procedimento iterativo até que todo vértice tenha uma lista cujo tamanho supere o número de vizinhos descoloridos. Acontece que isso é difícil de acontecer.

Antes de dizer o que queremos, precisamos de uma definição. Vamos denotar por $G_{i, c}$ o subgrafo de $G$ tal que $V\left(G_{i, c}\right)=V(G)$ e $u v \in E\left(G_{i, c}\right)$ se e somente se $u v \in E(G)$ e $c \in \mathcal{L}_{i}(u) \cap \mathcal{L}_{i}(v)$.

O que vamos fazer é chegar a uma iteração $i^{*}$ onde as listas de todos os vértices tenham tamanho pelo menos $\ell$ e, para toda cor $c$, o grafo $G_{i^{*}, c}$ tenha grau no máximo $\frac{\ell}{8}$. Nesse ponto, podemos aplicar o Teorema 12, que diz ser possível $\mathcal{L}$-colorir um grafo nessas condições.

\subsubsection{Parâmetros de interesse}

Para atingir esses objetivos, precisamos controlar dois parâmetros ao longo de todo o processo: 
i. $\ell_{i}(v)=$ tamanho da lista de cores do vértice $v$ no início da iteração $i$.

ii. $t_{i}(v, c)=$ número de vizinhos descoloridos de $v$ que possuem cor $c$ em suas listas no início da $i$-ésima iteração.

O nosso objetivo, em termos de $\ell_{i}$ e $t_{i}$, é chegar a uma situação onde existe um inteiro $\ell$ tal que $\ell_{i}(v) \geq \ell$ para todo $v$ descolorido e, para todo vértice descolorido $v$ e cor $c \in \mathcal{L}_{i}(v)$, temos $t_{i}(v, c) \leq \ell / 8$.

\subsubsection{Calculando algumas probabilidades}

Em vez de controlarmos os parâmetros $\ell_{i}(v)$ e $t_{i}(v, c)$ diretamente, vamos controlar apenas seus valores extremos $L_{i}$ e $T_{i}$. Estaremos interessados em que a seguinte propriedade valha com probabilidade positiva no começo de cada iteração $i$ :

Propriedade $\mathscr{P}_{i}$ : Para cada vértice descolorido $v$ e cor $c \in \mathcal{L}_{i}(v)$,

$$
\ell_{i}(v) \geq L_{i} \text { e } t_{i}(v, c) \leq T_{i}
$$

Vamos definir os valores de $L_{i}$ e $T_{i}$ previamente para todo $i \geq 1$, e depois disso, grande parte do trabalho consistirá em mostrar que a propriedade $\mathscr{P}_{i}$ vale durante um número suficiente de iterações para atingirmos o nosso objetivo. Ou seja, $\mathscr{P}_{i}$ vale pelo menos até que $T_{i} \leq L_{i} / 8$.

Antes de dar a definição de $L_{i}$ e $T_{i}$, considere, por um momento, uma situação idealizada onde, no começo de uma iteração $i$, para todo $v$ e cor $c \in \mathcal{L}_{i}(v)$, temos $\ell_{i}(v)=L_{i}$ e $t_{i}(v, c)=T_{i}$. Nesse caso, para cada cor $c \in \mathcal{L}_{i}(v)$, a probabilidade de que $c$ permaneça em $\mathcal{L}_{i+1}(v)$ após o passo 5 , isto é, de que $c$ não seja atribuída a nenhum vizinho de $v$ é:

$$
\mathbb{P}_{i}=\left(1-\frac{K}{\ln \Delta} \times \frac{1}{L_{i}}\right)^{T_{i}}
$$

Note que esta é também a probabilidade de que um vértice ativo retenha (após o passo 5) a cor que lhe foi atribuída. Um argumento parecido, porém um pouco mais complicado, mostra que a probabilidade de um vértice contado em $t_{i}(v, c)$ ser contado em $t_{i+1}(v, c)$ fica perto de $\left(1-\frac{K}{\ln \Delta} \times \mathbb{P}_{i}\right) \times \mathbb{P}_{i}$. Vamos fazer essa conta em detalhes na parte (b) da Afirmação 1 mais adiante.

Tipicamente, não é ideal a situação dos parâmetros $\ell_{i}(v)$ e $t_{i}(v, c)$. Ou seja, pode ser que $\ell_{i}(v)$ seja maior que $L_{i}$, e que $t_{i}(v, c)$ seja menor que $T_{i}$. 
Mas não é difícil calcularmos a verdadeira probabilidade de $c$ permanecer em $\mathcal{L}_{i+1}(v)$ após o passo 5 :

$$
\mathbb{P}_{i}(v, c)=\prod_{\substack{u \in \Gamma(v) \\ c \in \mathcal{L}_{i}(u)}}\left(1-\frac{K}{\ln \Delta} \times \frac{1}{\ell_{i}(u)}\right)
$$

Como podemos ver, é muito mais complicado lidar com um monte de probabilidades $\mathbb{P}_{i}(v, c)$ do que lidar com um único valor $\mathbb{P}_{i}$. É por isso que existe o passo 6 da iteração. As probabilidades $\mathbb{P}_{i, v, c}$ servem para uniformizar as probabilidades. Isto é, ao final do passo 6 a probabilidade de uma cor $c$ permanecer na lista de um vértice $v$ é $\mathbb{P}_{i}$ para todo $v$ e para toda cor $c \in \mathcal{L}_{i}(v)$. Precisamos dizer, então, quanto valem aquelas probabilidades $\mathbb{P}_{i, v, c}$. Ponha

$$
\mathbb{P}_{i, v, c}=1-\frac{\mathbb{P}_{i}}{\mathbb{P}_{i}(v, c)}
$$

Note que $\mathbb{P}_{i} \leq \mathbb{P}_{i}(v, c)$, e portanto a probabilidade acima está bem definida. Agora, a probabilidade de que uma cor $c \in \mathcal{L}_{i}(v)$ permaneça em $\mathcal{L}_{i+1}(v)$ após o passo 6 é $\mathbb{P}_{i}(v, c) \times\left(1-\mathbb{P}_{i, v, c}\right)=\mathbb{P}_{i}$, qualquer que seja $v$, e qualquer que seja $c \in \mathcal{L}_{i}(v)$.

Uma escolha natural para os valores de $L_{i+1}$ e de $T_{i+1}$ seria $L_{i+1}=$ $\min _{v}\left\{\mathbf{E}\left(\ell_{i+1}(v)\right)\right\}$ e $T_{i+1}=\max _{v, c}\left\{\mathbf{E}\left(t_{i+1}(v, c)\right)\right\}$. Infelizmente, não podemos provar que, com probabilidade positiva, para todo $v$ e para toda cor $c \in t_{i}(v, c)$, temos $\ell_{i+1}(v) \geq \mathbf{E}\left(\ell_{i+1}(v)\right)$ e $t_{i+1}(v, c) \leq \mathbf{E}\left(t_{i+1}(v, c)\right)$. Contudo, usando algumas ferramentas de concentração, somos capazes de provar que esses parâmetros ficam bem perto da média. Vamos definir os valores extremos desses parâmetros com uma pequena folga.

Colocamos $L_{1}=(1+\epsilon) \frac{\Delta}{\ln \Delta}, T_{1}=\Delta$, e definimos

$$
\begin{aligned}
& L_{i+1}=L_{i} \times \mathbb{P}_{i}-L_{i}^{2 / 3} \\
& T_{i+1}=T_{i} \times\left(1-\frac{K}{\ln \Delta} \times \mathbb{P}_{i}\right) \times \mathbb{P}_{i}+T_{i}^{2 / 3} .
\end{aligned}
$$

\subsubsection{Visão geral do restante da prova}

O que vamos fazer no restante da demonstração deste teorema é o seguinte. Vamos provar que $\mathbf{E}\left(\ell_{i+1}(v)\right)=\ell_{i} \times \mathbb{P}_{i}$ e que $\mathbf{E}\left(t_{i+1}(v, c)\right) \leq t_{i}(v, c) \times(1-$ $\left.\frac{K}{\ln \Delta} \times \mathbb{P}_{i}\right) \times \mathbb{P}_{i}$. Vamos provar que essas variáveis aleatórias são concentradas 
em torno da média. Vamos usar isso para mostrar que a propriedade $\mathscr{P}_{i}$ vale por um número suficiente de iterações. Vamos provar que, antes que a propriedade $\mathscr{P}_{i}$ passe a não valer mais, existe uma iteração $i^{*}$ tal que $T_{i^{*}} \leq L_{i^{*}} / 8$. Todas essas coisas são delicadas, por isso, tentaremos descrever e explicar todos os detalhes envolvidos em cada uma delas.

\section{Detalhes mais técnicos}

Surge um pequeno problema quando lidamos com o parâmetro $t_{i+1}(v, c)$. Na verdade, esse parâmetro não é fortemente concentrado em torno da média, pois ele pode cair para zero se $c$ é atribuído a $v$ na iteração $i$. Para contornarmos esse problema, vamos controlar um parâmetro ligeiramente diferente. Vamos chamar de $t_{i+1}^{\prime}(v, c)$ o número de vértices $u \in \Gamma(v)$ que são contados por $t_{i}(v, c)$ tais que no fim da iteração $i$ :

(i) permaneceram descoloridos,

(ii) nenhum vértice $w \in \Gamma(u) \backslash\{v\}$ recebeu cor $c$, e

(iii) $c$ não foi removido de $\mathcal{L}_{i+1}(u)$ no passo 6.

Note que, se $c$ não é atribuída a $v$, então $t_{i+1}^{\prime}(v, c)$ é idêntica a $t_{i+1}(v, c)$. Por outro lado, se $c$ é atribuída a $v$, então $t_{i+1}(v, c)=0$. Como estamos apenas querendo superestimar $t_{i+1}(v, c)$, isso não é problema.

Afirmação 1: Se $\mathscr{P}_{i}$ vale, então, para todo vértice $v$ e cor $c \in \mathcal{L}_{i}(v)$, temos:

(a) $\mathbf{E}\left(\ell_{i+1}(v)\right)=\ell_{i}(v) \times \mathbb{P}_{i}$;

(b) $\mathbf{E}\left(t_{i+1}^{\prime}(v, c)\right) \leq t_{i}(v, c) \times\left(1-\frac{K}{\ln \Delta} \times \mathbb{P}_{i}\right) \times \mathbb{P}_{i}+\frac{T_{i}}{L_{i}}$.

Prova. Para toda cor $c \in \mathcal{L}_{i}(v)$, a probabilidade de $c$ permanecer em $\mathcal{L}_{i+1}(v)$ é precisamente $\mathbb{P}_{i}$. A parte (a) segue da linearidade da esperança.

A menos que $c$ seja atribuído a $v$ na iteração $i$, temos que $t_{i+1}^{\prime}(v, c)=$ $t_{i+1}(v, c)$. Portanto, a probabilidade de que essas variáveis sejam diferentes 
é no máximo $\frac{K}{\ln \Delta} \times \frac{1}{L_{i}} \leq \frac{1}{L_{i}}$. Como $t_{i+1}^{\prime}(v, c)$ não pode exceder $T_{i}$, temos

$$
\begin{aligned}
\mathbf{E}\left(t_{i+1}^{\prime}(v, c)\right) & =\mathbf{E}\left(t_{i+1}^{\prime}(v, c) \mid v \text { recebeu cor } c\right) \times \operatorname{Pr}(v \text { recebeu cor } c) \\
& +\mathbf{E}\left(t_{i+1}^{\prime}(v, c) \mid v \text { não recebeu cor } c\right) \times \operatorname{Pr}(v \text { não recebeu cor } c) \\
& \leq T_{i} \times \frac{1}{L_{i}} \\
& +\mathbf{E}\left(t_{i+1}(v, c) \mid v \text { não recebeu cor } c\right) \times \operatorname{Pr}(v \text { não recebeu cor } c) \\
& =\frac{T_{i}}{L_{i}}+\mathbf{E}\left(t_{i+1}(v, c)\right) .
\end{aligned}
$$

Esse fato nos permite focar em $\mathbf{E}\left(t_{i+1}(v, c)\right)$ para estimar a esperança de $t_{i+1}^{\prime}(v, c)$. Existe um modo bem fácil, porém um pouco grosseiro, de se estimar $\mathbf{E}\left(t_{i+1}(v, c)\right)$. Para um vértice contado em $t_{i}(v, c)$ ser contado novamente em $t_{i+1}(v, c)$, uma das duas possibilidades abaixo deve ocorrer:

(i) ou $u$ não é ativado e $c$ permanece em sua lista,

(ii) ou $u$ é ativado, não mantém sua cor e $c$ permanece em sua lista.

Os dois eventos exigidos em (i) são independentes. Se os três eventos de (ii) fossem independentes, a probabilidade de um vértice contado em $t_{i}(v, c)$ ser contado novamente em $t_{i+1}(v, c)$ seria limitada superiormente por

$$
\left(1-\frac{K}{\ln \Delta}\right) \times \mathbb{P}_{i}+\frac{K}{\ln \Delta} \times\left(1-\mathbb{P}_{i}\right) \times \mathbb{P}_{i}=\left(1-\frac{K}{\ln \Delta} \times \mathbb{P}_{i}\right) \times \mathbb{P}_{i}
$$

Acontece que os eventos em (ii) não são independentes, e isso complica razoavelmente a análise das probabilidades. Vamos, então, tentar estimar a probabilidade de (ii).

Considere um vértice descolorido $u \in \Gamma(v)$ tal que $c \in \mathcal{L}_{i}(u)$. Para cada cor $d \in \mathcal{L}_{i}(u)-c$ vamos computar a probabilidade dos seguintes três eventos ocorrerem simultaneamente:

$A_{1}: c$ continuar em $\mathcal{L}_{i}(u)$,

$A_{2}$ : pelo menos um vizinho de $u$ ser ativado e colorido com cor $d$, e

$A_{3}: u$ ser ativado e receber cor $d$.

A probabilidade de (ii) ocorrer pode ser expressa como

$$
\sum_{\substack{d \in \mathcal{L}_{i}(u) \\ d \neq c}} \operatorname{Pr}\left(A_{1} \cap A_{2} \cap A_{3}\right)=\sum_{\substack{d \in \mathcal{L}_{i}(u) \\ d \neq c}} \operatorname{Pr}\left(A_{1} \cap A_{2} \mid A_{3}\right) \operatorname{Pr}\left(A_{3}\right) .
$$

Observe que os eventos $A_{1}$ e $A_{2}$ dependem unicamente das cores atribuídas aos vizinhos de $u$. Portanto, saber a cor que $u$ recebeu não dá absolutamente 
nenhuma informação sobre a ocorrência de $A_{1}$ ou sobre a ocorrência de $A_{2}$. Por essa razão, $\operatorname{Pr}\left(A_{1} \cap A_{2} \mid A_{3}\right)=\operatorname{Pr}\left(A_{1} \cap A_{2}\right)$. Vamos estimar $\operatorname{Pr}\left(A_{1} \cap A_{2}\right)$ através do cálculo de $\operatorname{Pr}\left(A_{2} \mid A_{1}\right)$.

Para qualquer vizinho $w$ de $v$ com $d \in \mathcal{L}_{i}(w)$, vamos considerar a probabilidade de $w$ ser ativado e receber cor $d$, dado que $A_{1}$ ocorreu. Acontece que essa é a mesma probabilidade de $w$ ser ativado e receber cor $d$, dado que $w$ não recebeu cor $c$. Pela definição de probabilidade condicional, isso é igual a

$$
\begin{aligned}
& \frac{\operatorname{Pr}(w \text { ser ativado e receber cor } d \mid w \text { não recebeu cor } c .)}{\operatorname{Pr}(w \text { não receber cor } c)} \\
= & \frac{\operatorname{Pr}((w \text { ser ativado e receber cor } d) \cap(w \text { não recebeu cor } c .))}{\operatorname{Pr}(w \text { não receber cor } c)} \\
\leq & \left(\frac{K}{\ln \Delta} \times \frac{1}{L_{i}}\right) /\left(1-\frac{K}{\ln \Delta} \times \frac{1}{L_{i}}\right) \\
= & \frac{K}{\ln \Delta} \times \frac{1}{L_{i}}+O\left(\frac{1}{L_{i}^{2} \ln ^{2} \Delta}\right) .
\end{aligned}
$$

Isto é verdade porque, se $0 \leq a \leq \frac{1}{2}$, então $\frac{a}{(1-a)} \leq a+2 a^{2}$. Agora, podemos calcular a probabilidade de $A_{2}$ acontecer dado que $A_{1}$ aconteceu. Essa probabilidade é no máximo

$$
1-\left(1-\frac{K}{\ln \Delta} \times \frac{1}{L_{i}}+O\left(\frac{1}{L_{i}^{2} \ln ^{2} \Delta}\right)\right)^{t_{i}(u, d)} \leq 1-\mathbb{P}_{i} .
$$

Se $u$ foi ativado e recebeu cor $c$, então é impossível $c$ continuar em $\mathcal{L}_{i+1}(u)$ e ao mesmo tempo $u$ ser descolorido. Então a probabilidade de $u$ continuar descolorido e $c$ permanecer em $\mathcal{L}_{i+1}(u)$ é no máximo

$$
\left(1-\frac{K}{\ln \Delta}\right) \times \mathbb{P}_{i}+\left(L_{i}-1\right) \times \operatorname{Pr}\left(A_{3}\right) \times \operatorname{Pr}\left(A_{1}\right) \times \operatorname{Pr}\left(A_{2} \mid A_{1}\right) .
$$

Isso é o mesmo que

$$
\left(1-\frac{K}{\ln \Delta}\right) \times \mathbb{P}_{i}+\frac{K}{\ln \Delta} \times\left(L_{i}-1\right) \times \frac{1}{L_{i}} \times \mathbb{P}_{i} \times\left(1-\mathbb{P}_{i}\right) .
$$

Finalmente, fazendo algumas pequenas contas, a probabilidade de que um vértice contado em $t_{i}(v, c)$ seja contado em $t_{i+1}(v, c)$ é no máximo

$$
\left(1-\frac{K}{\ln \Delta} \times \mathbb{P}_{i}\right) \times \mathbb{P}_{i}
$$


Pela linearidade da esperança, e pelo fato de que $\mathbf{E}\left(t_{i+1}^{\prime}(v, c)\right) \leq \mathbf{E}\left(t_{i+1}(v, c)\right)+$ $T_{i} / L_{i}$, temos que

$$
\mathbf{E}\left(t_{i+1}^{\prime}(v, c)\right) \leq t_{i}(v, c) \times\left(1-\frac{K}{\ln \Delta} \times \mathbb{P}_{i}\right) \times \mathbb{P}_{i}+\frac{T_{i}}{L_{i}}
$$

A demonstração da afirmação está completa.

Afirmação 2: Se para todo $j<i, L_{j}, T_{j} \geq \ln ^{7} \Delta$ e $T_{j} \geq \frac{1}{8} L_{j}$, então $T_{i} / L_{i}<$ $T_{i-1} / L_{i-1}$.

Prova. A prova é por indução em $i$. Com algumas contas, podemos checar que $T_{2} / L_{2}<T_{1} / L_{1}$. Vamos supor que a afirmação valha para todo $k \mathrm{com}$ $k \leq i$. Isso implica que $T_{i} / L_{i}<T_{1} / L_{1}<\ln \Delta$ e portanto $\mathbb{P}_{i} \geq \mathrm{e}^{-K T_{1} /\left(L_{1} \ln \Delta\right)}+$ $o(1)=\mathrm{e}^{-K(1+\epsilon)}+o(1)=\Omega(1)$. Por definição,

$$
L_{i+1}=L_{i}\left(\mathbb{P}_{i}-L_{i}^{-1 / 3}\right)
$$

Usando que $T_{i} \geq \ln ^{7} \Delta$ e que $\mathbb{P}_{i}=\Omega(1)$, temos

$$
\begin{aligned}
T_{i+1} & =T_{i} \times \mathbb{P}_{i}-T_{i} \times \frac{K \times \mathbb{P}_{i}^{2}}{\ln \Delta}+T_{i}^{2 / 3} \\
& \leq T_{i} \times \mathbb{P}_{i}-\left(K \times \mathbb{P}_{i}^{2}\right) \times T_{i}^{6 / 7}+T_{i}^{2 / 3} \\
& <T_{i} \times \mathbb{P}_{i}-T_{i}^{5 / 6} \\
& <T_{i} \times\left(\mathbb{P}_{i}-L_{i}^{-1 / 3}\right),
\end{aligned}
$$

Isso prova a afirmação.

Afirmação 3: Suponha que para todo $j<i, L_{j}, T_{j} \geq \ln ^{7} \Delta$ e $T_{j} \geq \frac{1}{8} L_{j}$. Então $\mathrm{e}^{-K(1+\epsilon)}+o(1) \leq \mathbb{P}_{i} \leq e^{-K / 8 \ln \Delta}$.

Prova. Na demonstração da afirmação anterior, já provamos o limitante inferior para $\mathbb{P}_{i}$. Para mostrar que vale o limitante superior, vamos olhar para a definição de $\mathbb{P}_{i}$ e para a hipótese de que $T_{j} \geq \frac{1}{8} L_{j}$.

$$
\mathbb{P}_{i}=\left(1-\frac{K}{\ln \Delta} \times \frac{1}{L_{i}}\right)^{T_{i}} \leq\left(1-\frac{K}{\ln \Delta} \times \frac{1}{8 T_{i}}\right)^{T_{i}} \leq \mathrm{e}^{-K / 8 \ln \Delta} .
$$


Lembre-se de que as escolhas aleatórias são do seguinte modo: primeiro escolhemos se vamos ou não ativar cada um dos vértices descoloridos; depois sorteamos uma cor para cada vértice ativo; e finalmente, para cada vértice descolorido $v$ e cor $c \in \mathcal{L}_{i}(v)$ que ainda não foi removida no passo 5 , jogamos uma moeda para decidir se $c$ continua ou não na lista de $v$.

Para aplicarmos a Desigualdade de Talagrand, os experimentos envolvidos devem ser feitos independentemente. Não é o que está acontecendo aqui porque alguns experimentos estão dependendo da saída de outros para serem executados. Por exemplo, sorteamos a cor de um vértice $v$ somente se o experimento "ativar vértice $(v)$ " deu sucesso.

Para contornar esse tipo de problema, vamos executar alguns experimentos "irrelevantes". Para cada vértice descolorido $v$, chame de $\operatorname{ATIVAR}(v)$ o experimento correspondente à ativação de $v$. Para cada vértice descolorido $v$, chame de $\operatorname{COR}(v)$ o experimento de sortear uma cor para $v$ (esteja $v$ ativo ou não). Finalmente, para todo vértice descolorido $v$ cor $c \in \mathcal{L}_{i}(v)$, chame de CARA-COROA $(v, c)$ o experimento de jogar uma moeda com probabilidade $\mathbb{P}_{i, v, c}$ de sucesso para decidir se excluímos a cor $c$ da lista de $v$ (mesmo que $c$ não pertença mais à lista de $v$ ). Vamos sortear uma cor para todo vértice, mesmo para aqueles que não foram ativados, e vamos lançar uma moeda para decidir se removemos $c$ da lista de $v$ para toda cor $c \in \mathcal{L}_{i}(v)$. É claro que a saída desses experimentos não tem efeito nenhum: não vamos atribuir nenhuma cor a um vértice inativo, e uma cor que não está mais na lista de $v$ não precisa ser removida.

Afirmação 4: Se $\mathscr{P}_{i}$ vale, $T_{i}, L_{i} \geq \ln ^{7} \Delta e T_{i} \geq \frac{1}{8} L_{i}$, então para todo vértice descolorido $v$ e cor $c \in \mathcal{L}_{i}(v)$, temos

(a) $\operatorname{Pr}\left(\left|\ell_{i+1}(v)-\mathbf{E}\left(\ell_{i+1}(v)\right)\right|>L_{i}^{2 / 3}\right)<\Delta^{-\ln \Delta}$;

(b) $S e t_{i}(v, c) \geq T_{i+1}$, então

$$
\operatorname{Pr}\left(\left|t_{i+1}^{\prime}(v, c)-\mathbf{E}\left(t_{i+1}^{\prime}(v, c)\right)\right|>T_{i}^{2 / 3}\right)<\Delta^{-\ln \Delta} .
$$

Prova. Vamos provar (a) primeiro. Para isso, é mais fácil mostrar que o número $\bar{\ell}$ de cores removidas da lista de um vértice $v$ é concentrado, do que mostrar que o número de cores que sobram na lista de $v$ é concentrado.

Se $u \in \Gamma(v)$, então uma alteração em $\operatorname{ATIVAR}(u)$ ou em $\operatorname{COR}(u)$ afeta o valor de $\bar{\ell}$ em no máximo 1 . Uma mudança em $\operatorname{ATIVAR}(u)$ ou em $\operatorname{COR}(u)$ não tem efeito nenhum sobre $\bar{\ell}$, se $u$ não for vizinho de $v$. Além disso, se mudarmos 
a saída de qualquer um dos experimentos $\operatorname{CARA-COROA}(v, c), c \in \mathcal{L}_{i}(v), \bar{\ell}$ é alterado de no máximo 1 . Seja $t$ um inteiro positivo qualquer, e suponha que $\bar{\ell} \geq t$. Para podermos aplicar a Desigualdade de Talagrand devemos ser capazes de achar um certificado curto para o fato de que $\bar{\ell} \geq t$. Sabemos que, para algum par de números $r$ e $s$, com $r+s=t$, existem $r$ vizinhos $u_{1}, \ldots, u_{r}$ de $v$, e $s$ cores $c_{1}, \ldots, c_{s} \in \mathcal{L}_{i}(v)$ tais que:

(i) $\operatorname{ATIVAR}\left(u_{i}\right)$ teve sucesso para todo $i \in[r]$,

(ii) $\operatorname{COR}\left(u_{i}\right) \neq \operatorname{COR}\left(u_{j}\right)$ para todo $i \neq j$,

(iii) $\operatorname{CARA}-\operatorname{COROA}\left(v, c_{i}\right)$ falhou para todo $i \in[s]$.

Esses experimentos certificam, portanto, que $\bar{\ell} \geq t$.

Agora estamos apenas a um passo de aplicar a Desigualdade de Talagrand. Resta verificar que $\mathbf{E}(\bar{\ell}) \geq L_{i}^{2 / 3}$, ou seja, que o desvio cuja probabilidade queremos limitar não é maior que a esperança da variável. Para provar isso vamos usar que $T_{i} \geq \frac{1}{8} L_{i}$, que $L_{i} \geq \log ^{7} \Delta$ e a cota para $\mathbb{P}_{i}$ fornecida pela Afirmação 3. Além disso, vamos usar também que $1-e^{-2 a} \geq a$ se $0<a<a_{0}$ para algum $a_{0}>0$. Assim, se $\Delta$ é suficientemente grande, temos que $K / 8 \ln \Delta$ é suficientemente pequeno para que a terceira desigualdade abaixo valha.

$$
\begin{aligned}
\mathbf{E}(\bar{\ell}) & \geq\left(1-\mathbb{P}_{i}\right) \times \ell_{i}(v) \geq\left(1-\mathrm{e}^{-K / 8 \ln \Delta}\right) \times L_{i} \\
& \geq \frac{K}{16 \ln \Delta} \times L_{i} \geq \frac{K}{16 L_{i}^{1 / 7}} \times L_{i} \geq L_{i}^{2 / 3} .
\end{aligned}
$$

Podemos, então, aplicar a Desigualdade de Talagrand como segue:

$$
\begin{aligned}
\operatorname{Pr}\left(|\bar{\ell}-\mathbf{E}(\bar{\ell})|>L_{i}^{2 / 3}\right) & <4 \mathrm{e}^{-\beta L_{i}^{4 / 3} / \mathbf{E}(\bar{\ell})} \\
& \leq 4 \mathrm{e}^{-\beta L_{i}^{4 / 3} / L_{i}} \\
& =4 \mathrm{e}^{-\beta L_{i}^{1 / 3}} \\
& \leq 4 \mathrm{e}^{-\beta \ln ^{7 / 3} \Delta} \\
& \leq \Delta^{-\ln \Delta}
\end{aligned}
$$

A passagem da $1^{\text {a }}$ para a $2^{\mathrm{a}}$ linha pode ser explicada da seguinte maneira. Sabemos que no início da iteração $i$ todas as listas têm tamanho pelo menos $L_{i}$, mas em nenhum momento da prova precisamos de que o tamanho dessas listas seja estritamente maior que $L_{i}$. Vamos supor, então, que as listas foram todas truncadas, no início da iteração $i$, para que tivessem todas tamanho exatamente $L_{i}$. Por isso, podemos usar tranqüilamente que $\mathbf{E}(\bar{\ell}) \leq L_{i}$. 
Pela linearidade da esperança temos $\mathbf{E}\left(\ell_{i+1}(v)\right)=\ell_{i}(v)-\mathbf{E}(\bar{\ell})$. Assim

$$
\begin{aligned}
& \operatorname{Pr}\left(\left|\ell_{i+1}(v)-\mathbf{E}\left(\ell_{i+1}(v)\right)\right|>L_{i}^{2 / 3}\right)= \\
& \operatorname{Pr}\left(\left|\ell_{i}(v)-\bar{\ell}-\left(\ell_{i}(v)-\mathbf{E}(\bar{\ell})\right)\right|>L_{i}^{2 / 3}\right)= \\
& \operatorname{Pr}\left(|\bar{\ell}-\mathbf{E}(\bar{\ell})|>L_{i}^{2 / 3}\right) \leq \Delta^{-\ln \Delta} .
\end{aligned}
$$

Agora vamos provar (b) decompondo a variável $t_{i+1}^{\prime}(v, c)$ em uma diferença de duas outras variáveis e provando que cada uma delas é concentrada. Chame de $T$ o conjunto de vizinhos de $v$ cujas listas contém $c$ no início da iteração $i$. Vamos definir $X$ como sendo o número de vértices em $T$ que permanecem descoloridos no final da iteração, e $Y$ ao número de vértices em $T$ que permanecem descoloridos e que tiveram a cor $c$ removida de suas listas por causa de um vizinho diferente de $v$ que recebeu cor $c$ ou porque o respectivo experimento CARA-COROA falhou.

Claramente $t_{i+1}^{\prime}(v, c)=X-Y$, e pela linearidade da esperança é suficiente mostrar que $X$ e $Y$ são concentradas o bastante. Vamos primeiro focar na variável $X$. Precisamos tomar cuidado aqui pois mudar a cor de $v$ pode alterar o valor de $X$ brutalmente. Para contornar esse problema, chame de $X_{1}$ o número de vértices em $T$ que receberam a mesma cor que um vizinho (diferente de $v$ ) ou que não foram ativados. Seja $X_{2}$ o número de vértices que receberam a mesma cor que $v$. Note que $X_{1}$ não é afetado pela cor de $v$. A seguir, vamos aplicar o Limitante de Chernoff para provar que o valor de $X_{2}$ é suficientemente pequeno. Depois vamos aplicar a Desigualdade de Talagrand para mostrar que $X_{1}$ é concentrada.

Vamos provar que $X_{2}$ é limitada por $\frac{1}{4} T_{i}^{2 / 3}$. Note que cada vértice de $T$ é ativado independentemente e recebe a mesma cor que $v$ com probabilidade no máximo $\frac{K}{\ln \Delta} \times \frac{1}{L_{i}}$. Como $|T| \leq T_{i}$, sabemos que $\bar{\ell}$ está limitado superiormente por uma binomial de parâmetros $T_{i}$ e $K / L_{i} \ln \Delta$ ). Temos então o seguinte:

$$
\operatorname{Pr}\left(X_{2}>\frac{1}{4} T_{i}^{2 / 3}\right) \leq \operatorname{Pr}\left(\operatorname{BIN}\left(T_{i}, \frac{K}{\ln \Delta} \times \frac{1}{L_{i}}\right)>\frac{1}{4} T_{i}^{2 / 3}\right)
$$

Pela Afirmação 2, sabemos que $\frac{T_{i}}{L_{i}}=\mathrm{O}(\ln \Delta)$. Portanto, $T_{i} \times \frac{K}{\ln \Delta} \times \frac{1}{L_{i}} \leq c$, para alguma constante $c$ independente de $\Delta$. Portanto, usando o Fato 1 
mencionado no Capítulo 4,

$$
\begin{aligned}
\operatorname{Pr}\left(\operatorname{BIN}\left(T_{i}, \frac{K}{\ln \Delta} \times \frac{1}{L_{i}}\right)>\frac{1}{4} T_{i}^{2 / 3}\right) & =\operatorname{Pr}\left(\operatorname{BIN}\left(T_{i}, \frac{c}{T_{i}}\right)>\frac{1}{4} T_{i}^{2 / 3}\right) \\
& \leq \frac{c^{T_{i}^{2 / 3} / 4}}{\left(T_{i}^{2 / 3} / 4\right) !} \\
& \leq \frac{1}{4} \Delta^{-\ln \Delta},
\end{aligned}
$$

quando $\Delta$ é suficientemente grande.

Agora vamos usar a Desigualdade de Talagrand para mostrar que $X_{1}$ é concentrada. Se $u \in T$, então uma alteração em $\operatorname{ATIVAR}(u)$ ou $\operatorname{COR}(u)$ afeta o valor de $X_{1}$ em no máximo 1 . Se $w \notin T$, uma mudança em $\operatorname{COR}(w)$ altera $X_{1}$ de no máximo 1 .

Se um vértice $u \in T$ não foi ativado, então a saída do experimento ATI$\operatorname{VAR}(u)$ certifica esse fato. Por outro lado, se $u$ foi ativado e foi descolorido por que um vizinho $w \neq v$ recebeu a mesma cor, então a saída dos experimentos $\operatorname{AtivaR}(w)$ e $\operatorname{COR}(w)$ e $\operatorname{COR}(u)$ certificam isso. Portanto, se $X_{1} \geq s$, existem no máximo $4 s$ experimentos cujas saídas certificam esse fato.

Para aplicarmos a Desigualdade de Talagrand precisamos, assim como na parte (a), checar se $\mathbf{E}\left(X_{1}\right)$ não é menor que o desvio cuja probabilidade queremos limitar. Em vez de provar que $\mathbf{E}\left(X_{1}\right) \geq \frac{1}{4} T_{i}^{2 / 3}$, vamos simplesmente observar que $\mathbf{E}\left(X_{1}\right) \geq \mathbf{E}\left(Y_{1}\right)$, onde $Y_{1}$ é uma das variáveis em que vamos decompor $Y$. A prova de que $\mathbf{E}\left(Y_{1}\right) \geq \frac{1}{4} T_{i}^{2 / 3}$ encontra-se mais abaixo, onde discutimos a concentração da variável $Y$.

Como $X_{1} \leq T_{i}$, temos que $\mathbf{E}\left(X_{1}\right) \leq T_{i}$ e a desigualdade de Talagrand implica

$$
\begin{aligned}
\operatorname{Pr}\left(\left|X_{1}-\mathbf{E}\left(X_{1}\right)\right|>\frac{1}{4} T_{i}^{2 / 3}\right) & <4 \mathrm{e}^{-\beta \frac{1}{16} T_{i}^{4 / 3} / T_{i}} \\
& =4 \mathrm{e}^{-\beta \frac{1}{16} T_{i}^{1 / 3}} \\
& \leq 4 \mathrm{e}^{-\beta \frac{1}{16} \ln ^{7 / 3} \Delta} \\
& \leq \frac{1}{4} \Delta^{-\ln \Delta} .
\end{aligned}
$$

Como $X_{1} \leq X \leq X_{1}+X 2$, esses dois limitantes juntos, mais o limitante de $X_{2}$ e o fato que $X_{1}$ é concentrada, implicam que

$$
\operatorname{Pr}\left(|X-\mathbf{E}(X)|>\frac{1}{2} T_{i}^{2 / 3}\right)<\frac{1}{2} \Delta^{-\ln \Delta} .
$$


Agora temos que provar que a variável $Y$ é concentrada. Vamos usar uma estratégia semelhante à que usamos para provar que $X$ é concentrada. Vamos considerar variáveis $Y_{1}$ e $Y_{2}$. A variável $Y_{2}$ é idêntica à variável $X_{2}$. A variável $Y_{1}$ conta o número de vértices de $T$ que:

(i) não são ativados ou são ativados e recebem a mesma cor que algum vizinho diferente de $v$,

(ii) a cor $c$ é removida de suas listas por causa de um vizinho diferente de $v$ que recebeu cor $c$ ou por causa de um experimento CARA-COROA mal sucedido.

Note que uma mudança na saída de $\operatorname{ATIVA}(v)$ ou $\operatorname{COR}(v)$ não afeta em nada o valor de $Y_{1}$. Se $u \in T$, uma mudança na saída de $\operatorname{ATIVA}(u), \operatorname{COR}(u)$ ou CARA$\operatorname{COROA}(u, c)$ pode alterar $Y_{1}$ de no máximo 1 . Finalmente, se $u \in \Gamma(T)-v$, mudando $\operatorname{ATIVA}(u)$ ou $\operatorname{COR}(u)$ também altera de no máximo 1 o valor de $Y_{1}$, pois $G$ tem cintura pelo menos 5 .

Suponha que $Y_{1}>s$. Para aplicar a Desigualdade de Talagrand, devemos ser capazes de exibir um certificado "pequeno" de que $Y_{1}>s$. No nosso caso, vamos exibir um certificado de tamanho no máximo $6 \mathrm{~s}$. Isso é feito do seguinte modo: para cada vértice $w$ de $T$ que é contado em $Y_{1}$, temos que certificar (i) e (ii). Para certificar (i), devemos exibir ATIVA $(w)$ e podemos parar por aí caso esse experimento tenha falhado. Se ATIVA $(w)$ sucedeu, então temos que exibir também $\operatorname{COR}(w), \operatorname{ATIVA}\left(w^{\prime}\right)$ e $\operatorname{COR}\left(w^{\prime}\right)$, onde $w^{\prime} \neq v$ é um vizinho de $w$. Para certificar (ii), temos que mostrar $\operatorname{CARA-COROA}(w, c)$ ou exibir $\operatorname{ATIVA}\left(w^{\prime \prime}\right)$ e $\operatorname{COR}\left(w^{\prime \prime}\right)$, onde $w^{\prime \prime} \neq v$ é um vizinho de $w$.

Se $\mathbf{E}\left(Y_{1}\right) \geq \frac{1}{4} T_{i}^{2 / 3}$, então podemos aplicar a Desigualdade de Talagrand. Vamos então provar que a esperança de $Y_{1}$ não é menor que $\frac{1}{4} T_{i}^{2 / 3}$. Usando 
técnicas semelhantes àquelas utilizadas para limitar a esperança de $\bar{\ell}$, temos

$$
\begin{aligned}
\mathbf{E}\left(Y_{1}\right) & \geq\left(1-\mathbb{P}_{i}\right) \times \mathbf{E}\left(X_{1}\right) \\
& \geq\left(1-\mathbb{P}_{i}\right) \times\left(|T| \times\left(1-\frac{K}{\ln \Delta}\right)-\mathbf{E}\left(X_{2}\right)\right) \\
& \geq\left(1-\mathrm{e}^{-K / 8 \ln \Delta}\right) \times\left(\frac{1}{2} T_{i+1}-c\right) \\
& \geq\left(1-\mathrm{e}^{-K / 8 \ln \Delta}\right) \times \frac{1}{4} T_{i+1} \\
& \geq \frac{K}{16 \ln \Delta} \times \frac{1}{4} T_{i} \times\left(1-\frac{K}{\ln \Delta} \times \mathbb{P}_{i}\right) \times \mathbb{P}_{i} \\
& \geq \frac{K}{64 T_{i}^{1 / 7}} \times T_{i} \times \frac{1}{2} \mathrm{e}^{-K(1+\epsilon)} \geq \frac{1}{4} T_{i}^{2 / 3}
\end{aligned}
$$

Finalmente, podemos aplicar Talagrand para mostrar que $Y_{1}$ é uma variável aleatória concentrada.

$$
\begin{aligned}
\operatorname{Pr}\left(\left|Y_{1}-\mathbf{E}\left(Y_{1}\right)\right|>\frac{1}{4} T_{i}^{2 / 3}\right) & <4 \mathrm{e}^{-\beta \frac{1}{16} T_{i}^{4 / 3} / T_{i}} \\
& =4 \mathrm{e}^{-\beta \frac{1}{16} T_{i}^{1 / 3}} \\
& \leq 4 \mathrm{e}^{-\beta \frac{1}{16} \ln ^{7 / 3} \Delta} \\
& \leq \frac{1}{4} \Delta^{-\ln \Delta} .
\end{aligned}
$$

Novamente, como $Y_{1} \leq Y \leq Y_{1}+Y_{2}$, segue que

$$
\operatorname{Pr}\left(|Y-\mathbf{E}(Y)| \geq \frac{1}{2} T_{i}^{2 / 3}\right)<\Delta^{-\ln \Delta} .
$$

Agora, juntando 6.1 e 6.2, obtemos a cota desejada em (b):

$$
\operatorname{Pr}\left(\left|t_{i+1}^{\prime}(v, c)-\mathbf{E}\left(t_{i+1}^{\prime}(v, c)\right)\right|>T_{i}^{2 / 3}\right)<\Delta^{-\ln \Delta} .
$$

A demonstração da afirmação está completa.

Afirmação 5: Se para todo $1 \leq j<i, L_{j}, T_{j} \geq \ln ^{7} \Delta$ e $T_{j} \geq \frac{1}{8} L_{j}$, então $\mathscr{P}_{i}$ vale com probabilidade positiva.

Prova. Vamos provar a afirmação por indução em $i$. A propriedade $\mathscr{P}_{1}$ vale. Suponha que $i>1$ e que $\mathscr{P}_{i-1}$ valha. Vamos provar que com probabilidade positiva $\mathscr{P}_{i}$ vale. 
Para todo vértice $v$, considere o evento $A_{v}$ em que $\ell_{i+1}(v)<L_{i+1}$, e para todo vértice $v$ e cor $c \in \mathcal{L}_{i}(v)$, chame de $B_{v, c}$ o evento em que $t_{i+1}(v, c)>$ $T_{i+1}$. Vamos mostrar, usando o Lema Local, que com probabilidade positiva nenhum desses eventos ruins ocorrem. E isso é suficiente para mostrar que $\mathscr{P}_{i-1}$ vale. Observe que só temos interesse em calcular $t_{i+1}(v, c)$ para vértices $v$ que permaneceram descoloridos após a iteração $i$. E nesses casos, $t_{i+1}(v, c)=t_{i+1}^{\prime}(v, c)$. Portanto, se algum evento $B_{v, c}$ ocorre, então $\left|t_{i+1}^{\prime}(v, c)-\mathbf{E}\left(t_{i+1}^{\prime}(v, c)\right)\right|>T_{i}^{2 / 3}$.

Pela Afirmação 4, todos esses eventos têm probabilidade no máximo $\Delta^{-\ln \Delta}$. Além disso, cada evento $A_{v}$ (e cada evento $B_{v, c}$ ) depende dos experimentos $\operatorname{ATIVA}(u), \operatorname{COR}(u)$ e $\operatorname{CARA}-\operatorname{COROA}(u, d)$ somente quando o vértice $u$ está distante no máximo 2 de $v$. Assim, cada evento ruim é mutuamente independente de todos os eventos ruins exceto um número limitado deles. $\mathrm{O}$ número de eventos dependentes de um particular evento ruim não passa de $d=\Delta+\Delta^{2}+\Delta^{3}+\Delta^{4}+\left(\Delta+\Delta^{2}+\Delta^{3}+\Delta^{4}\right) \times(1+\epsilon) \frac{\Delta}{\ln \Delta}<\Delta^{5}$ para $\Delta$ suficientemente grande. Novamente, se $\Delta$ é suficientemente grande, então $4 \times \Delta^{5} \times \Delta^{-\ln \Delta}<1$, e pelo Lema Local, com probabilidade positiva, nenhum dos eventos que queríamos evitar ocorre. Isso prova a afirmação.

Para que seja possível verificar a integridade da demonstração, vamos enunciar o resultado que falta, porém não vamos demonstrar. A prova desse último resultado consiste, fundamentalmente, em estimar precisamente qual é o decaimento da relação $T_{i} / L_{i}$. Ela pode ser encontrada em [12].

Afirmação 6: Existe um índice $i^{*}$ tal que

(a) Para todo $i \leq i^{*}, T_{i} \geq \log ^{8} \Delta, L_{i}>\Delta^{\epsilon / 3}$, e $T_{i} \geq \frac{1}{8} L_{i}$;

(b) $T_{i^{*}+1} \leq L_{i^{*}+1}$.

Com as afirmações 5 e 6 , a prova do teorema está completa. 


\section{Referências Bibliográficas}

[1] Noga Alon, Restricted colorings of graphs, Surveys in combinatorics, 1993 (Keele), London Math. Soc. Lecture Note Ser., vol. 187, Cambridge Univ. Press, Cambridge, 1993, pp. 1-33. MR 94g:05033

[2] Noga Alon and Joel H. Spencer, The probabilistic method, second ed., Wiley-Interscience Series in Discrete Mathematics and Optimization, Wiley-Interscience [John Wiley \& Sons], New York, 2000, With an appendix on the life and work of Paul Erdős. MR 2003f:60003

[3] Tom Bohman and Ron Holzman, On a list coloring conjecture of Reed, J. Graph Theory 41 (2002), no. 2, 106-109. MR MR1926312 (2003h:05078)

[4] Béla Bollobás, Modern graph theory, Springer-Verlag, New York, 1998. MR 99h:05001

[5] O. V. Borodin and A. V. Kostochka, On an upper bound of a graph's chromatic number, depending on the graph's degree and density, J. Combinatorial Theory Ser. B 23 (1977), no. 2-3, 247-250. MR MR0469803 (57 \#9584)

[6] Paul A. Catlin, A bound on the chromatic number of a graph, Discrete Math. 22 (1978), no. 1, 81-83. MR MR522914 (80a:05090a)

[7] A. Johansson, Asymptotic choice number for triangle free graphs, DIMACS Technical Report (1996), 91-5.

[8] Jeong Han Kim, On Brooks' theorem for sparse graphs, Combin. Probab. Comput. 4 (1995), no. 2, 97-132. MR MR1342856 (96f:05078)

[9] Jim Lawrence, Covering the vertex set of a graph with subgraphs of smaller degree, Discrete Math. 21 (1978), no. 1, 61-68. MR MR523419 (80a:05094) 
[10] László Lovász, Combinatorial problems and exercises, second ed., NorthHolland Publishing Co., Amsterdam, 1993. MR 94m:05001

[11] Michael Molloy and Bruce Reed, A bound on the total chromatic number, Combinatorica 18 (1998), no. 2, 241-280. MR 99j:05074

[12] Graph colouring and the probabilistic method, Algorithms and Combinatorics, vol. 23, Springer-Verlag, Berlin, 2002. MR 2003c:05001

[13] Kyoji Ohba, On chromatic-choosable graphs, J. Graph Theory 40 (2002), no. 2, 130-135. MR MR1899118 (2003b:05069)

[14] Bruce Reed, The list colouring constants, J. Graph Theory 31 (1999), no. 2, 149-153. MR MR1686286 (2000c:05070)

[15] Bruce Reed and Benny Sudakov, List colouring of graphs with at most $(2-o(1)) \chi$ vertices, Proceedings of the International Congress of Mathematicians, Vol. III (Beijing, 2002) (Beijing), Higher Ed. Press, 2002, pp. 587-603. MR MR1957563 (2003m:05078)

[16] L List colouring when the chromatic number is close to the order of the graph, Combinatorica 25 (2005), no. 1, 117-123. MR MR2109199

[17] Sheldon Ross, A first course in probability, 6th ed., Prentice Hall, 2001.

[18] V. G. Vizing, Some unsolved problems in graph theory, Uspehi Mat. Nauk 23 (1968), no. 6 (144), 117-134. MR MR0240000 (39 \#1354) 